\title{
ZUR VORGESCHICHTE DES REINKE VOS.
}

In mehr als einem punkte ist die vor- und entstehungsgeschichte des Reinke Vos noch unaufgeklärt; zwar haben in den letzten jahrzehuten die gemeinsamen bemühungen niederlïndischer und deutscher forscher dieselbe im allgemeinen klargestellt und vor allem erwiesen, dass unser gedicht kein original ist, allein in einzelnen durfte sich noch manches finden, was einer nachprufung und richtigstellung bedarf. Hierzu beizutragen soll die aufgabe der nachfolgenden seiten sein.

Bei der frage nach dem verhältnis des R. V. zu seiner niederländischen quelle hat man bisher, wenn man von einigen gelegentlichen bemerkungen absieht, allzusehr den erzählungstext, zu wenig die glosse berücksichtigt; selbst Lubben, dessen verdienst es ist, endlich einmal die sogenannte katholische glosse wider abgedruckt zu haben, schweigt in der einleitung zu seiner ausgabe uber ihr verhältnis zur quelle. Ich werde dieselbe, nachdem die dem R. V. zunächst liegende nl. uberlieferung besprochen und vorher über den verfasser des glossierten und mit capiteluberschriften versehenen Reinaert gehandelt worden ist, zusammen mit der äussern einteilung einer eingebenden vergleichung mit dem nl. unterziehen und zum schluss das verhältuis des ganzen R. V. zum niederlăndischen betrachten.

Im verlaufe meiner arbeit habe ich mich, im anschluss an Martin, ausgabe des Reinaert, Paderborn 1874, folgender abkürzungen bedient:

a (od. R. I) = ältestes nl. gedicht von Willem (ComburgStuttgarter hs.); ich citiere nach Martins ausgabe s. 1-105.

b (od. R. II) $\doteq$ umarbeitung von a mit der fortsetzung (Amsterdam-Brüsseler hs.); bei Martin s. 107-341. 
c = Haager (v. Wijnsches) bruchstlick; die varianten bei Martin von v. 6761 an.

$\mathrm{d}=$ bruchstuck eines gedruckten gereimten Reinaert veroffentlicht von Culemann und danach von Hoffmann v. Fallersleben in seinen Horae Belgicae XII, S. 7-15.

$\mathrm{h}=$ das $\mathrm{nl}$. volksbuch Reynaert de Vos Antwerpen 1564, hrsgb. v. Martin, Paderborn 1877.

$1=$ lateinische, vor 1280 von Balduin verfasste tibersetzung von $R$ I, nach dem druck von 1473 hrsgb. v. M. F. A. G. Campbell, Haag 1859 und danach von W. Knorr, Eutin 1860. $\mathrm{p}=$ Die historie vā reynaert de vos (prosa-auszug). Nach der ausg. Delft 1485 hrsgb. v. L. Suhl, Liubeck 1783.

$\mathbf{r}=$ Reinke de Vos. Nach d. ausg. Lübeck 1498 hrsgb. v. Lubben, Oldenburg 1867.

\section{Hinrek van Alckmer.}

Seitdem durch Jakob Grimm (Reinhart Fuchs s. CLXXV fr.) die törichte ansicht, dass der Nieder länder Hinrek van Alckner der verfasser des niederdeutschen R. V. sei, abgewiesen und der angabe der ersten vorrede des R. V. (Lubben s. III u. IV) die einzig richtige und mögliche erklärung gegeben worden ist, 'dass Heinrich, etwa 100 jahre später die niederländischen gedichte einer gelinden umarbeitung unterzog, die sich wol gar auf beifügung der vorrede und einteilung in bucher und capitel beschränkte', hat man zu weiterer aufklärung liber die person des H. v. A. die blicke nach den Niederlanden gerichtet und dort den veranstalter der betreffenden uberarbeitung in dem von Scheltema (Reintje de Vos Haarlem 1826 s. XXIX d. einltg.) aus $K$. Burmans utrechtschen jaarboeken III, 183. 373 in urkunden von 1477 und 1481 nachgewiesenen Hendrik van Alkmaar finden zu können geglaubt. Jakob Grimm selbst (a. a. o. s. CLXXVI) ist es gewesen, der nach einer andeutung Scheltemas (a. a. o. s. XXX) die verınutung weiter ausführte, dass dieser H. v. A., weil er später in Utrecht nicht weiter auftritt, mit Philippa von Egmont, tochter des herzogs Adolf von Geldern, die sich 1485 dem herzog v. Lothringen Renat II. vermählte, in lothringische dienste gieng und den seit 1486 geborenen herzoglichen kindern als lehrer zugegeben wurde; 
'leicht konnte Renat, schliesst Grimm, seiner niederländischen gemahlin $\mathrm{zu}$ liebe, die söhne in dieser sprache unterweisen lassen, und die neue bearbeitung des beribmten werks wunschen. Solche vermutungen bedürfen noch grösserer sicherheit; Heinrich mlisste um 1490 oder bald nachher hand angelegt haben und Baumann seinen fussstapfen beinahe unmittelbar gefolgt sein'. - Die von Grimm für seine vermutung verlangte grössere sicherheit bin ich zu geben nicht im stande, muss vielmehr seinen letzten worten widersprechen, indem mir keine andere auffassung derselben möglich erscheint als die, dass H. v. A. die im auftrag des herzogs veranstaltete bearbeitung des Reinaert dem unterricht der prinzen in der nl. sprache zu grunde gelegt hätte. - Die beiden ersten kinder aus jener ebe, Charles geboren 1486, François geboren 1487, sind in zartem alter gestorben (s. Don Calmet, histoire eccles. et civile de Lorraine II, 1118). Dass Renat II. schon vor oder bei der geburt eines dieser söbne dem H. v. A. zur bearbeitung eines Reinaert sollte auftrag gegeben haben, um jene später danach unterrichten zu lassen, ist doch recht unwahrscheinlich und wir wirden, weun wir Grimms letzte worte annehmen, zu dieser ansicht gezwungen sein, da Henriks exemplar schon 1487 bei Leeu erschien (s. u. s. 9): Renat sorgte zwar ausserordentlich gut fir die erziehung seiner söhne ${ }^{1}$ ), aber solche sorgfalt, die sich sogar mehrere jahre vor dem beginn des eigentlichen unterrichts auf die auswahl des stoffes im einzelnen erstreckt bätte, wäre doch allzu seltsam und voreilig gewesen. Der nächst geborene soln Antoine, der den eltern nicht so jung entrissen wurde und dem der vater unter der leitung des gelehrten Philibert de Stainville eine äusserst gewissenhafte erziehung zu teil werden liess'), wurde erst am 4. juni 1489 geboren (Don Caluet II, 1118) und vom 7. jahre an unterrichtet - das sind ziffern, welche diesseits des druckjahres

1) Don Calmet a. a. o. II, 1127: 'Depuis l'âge de sept ans, le jeune Prince (Antoine) fut élevé avec grand soin par le duc René, qui lui donna pour gouverneur un très sage vieillard, âgé d'environ quatrevingts ans, nommé Philibert de Stainville. Philibert avoit sous lui d'autres maîtres de toutes sortes, pour former le jeune prince dans les lettres, dans la religion, et dans tous les exercices conformes à son condition' etc. 
von d liegen. Auch darf man wol fragen, ob denn gerade der Reinaert mit seinen die unsittlichkeit freilich strafenden, aber daher doch auch dieselbe aufdeckenden stellen für die erziehung eines kindes die geeignete grundlage abgeben konnte.

Wollen wir daher Grimms vernutung nicht aufgeben ${ }^{1}$ ), so werden wir die worte der ersten vorrede im R. V. (s. III u. IV) 'scholemester unde tuchtlerer des eddelen dogentliken vorsten unde heren, hertogen van Lotringen' und 'umme bede willen mines gnedigen heren' so verstehen müssen, dass $\mathrm{H}$. v. A. den herzog selbst in der nl. sprache unterrichtet und zu diesem zweck den Reinaert nur bearbeitet habe. Von der verheiratung Renats $14 \delta 5$ bis zur ausgabe des buches 1487 war dic bearbeitung leicht getan, - möglicherweise hatte aber schon vorher Heinrich sein manuscript fertig und gab es erst auf veranlassung des herzogs zum druck: die bede, die der herzog an H. v. A. richtete, würde dann auf das interesse zurtick zu fuhren sein, welches derselbe im täglichen verkehr mit seiner gemahlin fur deren sprache leicht gewinnen konnte und was war natürlicher, als dass er bei erlernung der fremden sprache sich nicht bloss auf die unterhaltung mit der herzogin beschränkte, sondern weitere aufklärung von dem scholemester verlangte? Zeitlich steht dem nichts entgegen, ja, es wäre vielleicht die einzige möglichkeit, H. v. A. mit Renat II. zusammen zu bringen; denn die bearbeitung von d liegt nach dem erscheinen der Goudaer prosa 1479 (s. u. s. 52) sowie des Dialogus creaturarum 1480 (s. u. s. 29) und Renat hatte von diesem jahre bis zu seiner verheiratung, durch die er uberhaupt erst in verbindung mit den Niederlanden trat, keine ersichtliche veranlässung, niederländisch zu lernen.

Ein anderer Henrik van Alkmaar ist an etwas verstecktem orte in den jahren 1457-1478 nachgewiesen. Delprat nämlich

1) - und sie hat noch immer die meiste wahrscheinlichkeit fïr sich; denn Nieder-Lothringen, an das man versucht sein könnte zu denken, und dessen herzöge selbst nach der vereinigung mit Brabant noch lange den titel dux Lotharingiae fortführten, hatte in der uns angehenden zeit regenten, die nicht einfach 'herzog von Lothringen' genannt werden konnten: weder Karl der kühne v. Burgund (146i-14i6) noch Maria, gemahlin von Maximilian v. Oesterreich (1476-1482) noch der erst 1478 geborene Philipp v. Oesterreich (1482-1506). 
machte in dem im 6. teile von Kist en Royaards Archief voor kerkelijke Geschiedenis, Leiden 1835 enthaltenen aufsatz 'Verslag omtrent eenige Handschriften, in de koninklijke Haagsche Boekerị berustende, meest betrekkelijk de Fraterhuizen' den inhalt einer papierhandschrift bekannt, welche 65 bll. in $4^{0}$ umfasst und offenbar im 15. jahrhundert geschrieben ist (jetzt bezeichnet: Bibl. reg. Hagan. cod. manscr. no. 346). Der verfasser nennt sich in der uberschrift: 'Incipit narratio de inchoatione status nostri et deinde de fratribus huius domus nostre auctore domino Jacobo traiectensi alias Voecht seniore nro. lxxx $x^{\text {ario' }}$ und den inhalt der hs. charakterisiert Delprat (a. a. 0. s. 278): 'Men vindt hier een verbaal van de merkwaardigste voorvallen en eene levensgeschiedenis der personen, behoord hebbende tot het zoogenaamde Rijke Fraterhuis in $\mathrm{Zwol}$, gesticht ten jahre 1394 in de nabijheid van het Oude of Arme Fraterhuis'.

Uns gehen aus der hs. zwei stellen an; die erste steht bei Delprat a. a. o. s. 291: 'De transitu eius (sc. Theodorici van Herxen) felici ex hac vita. Transiit anno 1457, aetatis ut arbitror 76, regiminis domus nostrae 47. In sepultura fiebat magnus concursus clericorum'; wozu Delprat bemerkt: ' $\mathrm{Bij}$ deze gelegenheit treft men de lijst aen van al destijds levende inwoners van het Fraterhuis ... Wel lest men hier den naam van Henricus Alkmariae onder de genen, welke de uitvaart van Diedrik van Herxen bijwoonden.' - Die zweite stelle lautet bei Delprat a. a. 0. s. 295: 'De dmno Henrico Alcmariae [Zoo lang hij onder de Broeders verkeerde, 'custos horologii et confessor sororum' van het klooster op den Maet onder 'Zwol].'

Delprat vermutet nun a. a. o. a. $292 \mathrm{ff}$, dass Philippa von Egmont, gemahlin von René II. von Lotbringen, diesem Heinrich v. Alkmaar den unterricht ihrer kinder anvertraut habe und hält es für möglich, dass $H$. v. A. der verfasser des R. V. sei, indem er s. 293 sagt: 'Indien Henrik van Alkmaer in het jaer 1457 , in den ouderdom van 20 jaer, bij de uitvaart van Diederik van Herxen is tegenwordig geweest, zal hij in den ouderdom van 61 jaer de bekende vertaling van Reintje de Vos, in 1498 hebben kunnen uitgeven.' Diese vermutung fällt von selbst: einmal weil sie den Niederländer zum verfasser eines 
niederdeutschen werkes hinstellt und dann weil dieser H. v. A., der doch sicher an beiden stellen eine und dieselbe person ist, uberhaupt gar nicht von Philippa als herzogin von Lothringen berufen werden konnte, da er schon vor 1478 gestorben war (s. u. s. 7). - Damit ist auch das urteil ausgesprochen uber Ulmanns annabme dieser vermutung, welcher (Reformatoren vor der reformation, Hamburg 1842, II, s. 300) hier im Fraterhause zu Zwoll den Heinrich von Alkmar leben lässt, 'dem die nl. bearbeitung des Reinecke Fuchs zugeschrieben wird, eines gedichts, dessen humoristische polemik gegen den clerus und manche kircheneinrichtungen $\mathrm{zu}$ der tendenz der freisinnigen brider vom gemeinsamen leben wol passen würde' und es (a. a. o. anm. 5) für merkwürdig hält 'die imitatio Christi und den Reinecke Fuchs auf dem nämlichen boden zu finden, die, so himmelweit verschieden sie sind, doch darin tibereinkommen, dass ihr geist dem äusserlichen wesen in der frönmigkeit entgegen gesetzt ist, nur auf der einen seite mit tiefernster innerlichkeit, auf der andern mit heiterem spott.'

Wenn nun aber auch diese combinationen als unzulässig abgewiesen werden müssen, so dürfen wir doch diesem Henricus Alcmariae die beachtung nicht von vornherein versagen und besonders die letzte der beiden oben angegebenen stellen ist wol wert, ganz mitgeteilt zu werden; hierzu bin ich durch herrn oberbibliothekar dr. M. F. A. G. Campbell im Haag in stand gesetzt, dem ich für diese wie für andere bereitwilligst erteilte auskunft auch an dieser stelle meinen herzlichsten dank ausspreche. Die stelle steht auf fol. $43^{\text {b }}$ der erwähnten hs. und lautet: 'De domino henrico alcmarie fratre nostro. Post hunc (sc. Arnoldum de Vollenhoe) fuit dilectus frater noster dominus henricus alcmarie qui erat aliqualiter longe stature sed macer. Ipse bene exercitatus et maturus in moribus et studiosus in scripturis et multa copulavit [compilavit?] strenuus in exerciciis secundum morem antiquum. Scriptor bonus in bastardo et ad tempus rasor et custos horologii et oratorii. Factus autem sacerdos non longe post ordinatus est confessor sororum op die maet. quas cum magna diligentia regens et custodiens satis multos dolores sustinuit propter eas cum domus illa adhuc nondum plene purgata fuisset a malo regimine primi rectoris eius. Ipse ergo in regimine earum existens sepissime 
venit al fratres nostros. quem a puero usque in finem innocentie iura observantem dominus properavit educere de medio iniquitatum. Nam dum pestis apud nos regnaret ipse sepe a domo sua ter maet venit ad nos in clusam afferens cuneos vel simile et prandebat nobiscum. Semel ergo rediens a nobis statim peste tactus decubuit in domo nostro nomine melthuys. puto quinta vel sexta die infirmitatis sue que fuit dies sancti Jacobi apostoli dixit Sancte iacobe ora pro me et sic expiravit. appositus ad patres et fratres suos et sepultus in windesim in ordine fratrum.

De domino rutghero de doetenghen fratre nostro' etc.

Dieser bruder Rutgherus de Doetenghen starb, wie später erzïhlt wird, 1478. Heinrich von Alkmaar folgte auf Arnoldus de Vollenhoe und dieser auf Jacobus Goch, welcher 1472 starb; also ist Heinrich von Alkmaar nach 1472 und vor 1478 gestorben.

Ist es nun möglich, dass dieser H. v. A. der uberarbeiter des ul. Reinaert gewesen wäre? Wir glauben diese frage mit nein beantworten $\mathrm{zu}$ mussen und gehen dabei von der sicher zutreffenden voraussetzung aus, dass der hier und oben bei dem leichenbegängnis des Dietrich v. Herxen erwähnte H. v. A. eine und dieselbe person ist. Jacob Voecht wusste von ihm von seiner jugend an ('quem a puero usque in finem innocentie iura observantem'), schildert ihn in seiner weise genau und wïrde es gewiss nicht unterlassen haben zu berichten, wenn er auch literarische tätigkeit entfaltet hätte oder wenn er gar auf einige zeit aus dem stillen leben des Fraterhauses zum unterricht eines lothringischen prinzen wäre abgerufen worden. Es wird nur von ihm gesagt, dass er in der gewöhnlichen schrift ('bastardo', vgl. Wattenbach, Schriftwesen im Mittelalter s. 284) ein guter schreiber gewesen sei und dass er sich eifrig mit dem schreiben von btichern abgegeben habe ('studiosus in scripturis'). Aus dem ganzen geht hervor, dass er ein zu eifriger bruder war (rgl. auch 'multos dolores sustinuit'), als dass er sich mit abfassung von profanen werken hätte abgeben können. Auch würde die vollendung des werkes vor 1478 fallen, womit wir eine zeit erreichen wurden, in welcher (s. o. s. 4) d nicht entstanden sein kann. 


\section{Die Culemannschen bruchstücke.}

Die auffindung von bruchstlucken eines gedruckten nl. gedichts von Reinaert durch senator Culemann in Hannover und deren veröffentlichung durch Hoffmann v. Fallersleben in den Hor. Belg. XII, 7 ff. nach dem von Culemann veranstalteten getreuen abdruck brachte neue aufschlusse uber die abhängigkeit des R. V. vom niederländischen. Allein eine durchaus sichere vergleichung derselben mit dem $R$. V. liess sich nicht vornehmen, da Hoffmanns abdruck ungenau ist und der wichtigkeit nicht ausreichend rechnung trägt, welche die bruchstäcke fur die vorgeschichte des R. V. haben. Ganz abgesehen davon, dass Hoffmann in seiner uniformierenden weise die orthographie änderte und nichts mitteilte uber anfang und ende der blätter, etwaige custoden und seitenziffern, stellung der holzschnitte u. s. w. hat er vor allen dingen ganze wörter und zeilen des alten druckes ausgelassen. Durch die gute des herrn G. A. S. Schneider, B.-A. in Cambridge, dem ich für seine mannigfachen mubleistungen $\mathrm{zu}$ aufrichtigem danke verbunden bin, ist es mir möglich, einen buchstaben- und zeilengetreuen abdruck $^{1}$ ) des originals sowie die nötigen mitteilungen uber dasselbe zu geben. Es sind 7 bll. in $4^{0}$; sie haben, soweit sie ganz erhalten sind, die böhe und breite des bei Hoffmann reproducierten bolzschnittes. Seitenziffern und custoden fehlen. Bl. 2 trägt unten am rande rechts von vers 71 die signatur hiij. Vollständig erhalten sind bl. 5 und bl. 7, welche noch spuren der bll. zeigen, mit denen sie einst zusammenhiengen. Bl. 2 darf man füglich auch zu den vollständig erhaltenen rechnen: die erste zeile desselben ( $v .42^{b}$ - bei Hoffmann als fehlend bezeichnet) ist zwar durchschnitten, lässt aber noch die worte Niel wt en mochte daar hi inq... lesen.

Von bl. 1, welches mit bl. 3 zusammenhängt, sind, wie aus einer vergleichung mit bl. $5^{a}$ und $7^{\text {a }}$ hervorgeht (und ausserdem der text lehrt, welcher $b$ folgt) oben 4 zeilen abgeschnitten; ebenso von bl. 3 , welches ausserdem an der seite in der weise verstummelt ist, dass zwar der text auf der stirnseite bis auf einige buchstaben erhalten ist, jedoch von der uberschrift

1) S. u. s. $10-16$. 
mehrere buchstaben fehlen, - dem entsprechend zeigt der holzschnitt auf der rilckseite des bl. 3 defecte stellen.

Bl. 4 bildet mit bl. 6 ein doppelblatt und ist in derselben weise beschnitten, wie die eben beschriebenen bll. 1 und 3, so dass also vor v. 90 auf bl. $4^{\text {b }} 4$ zeilen fehlen und demgemäss der holzschnitt auf bl. $4^{\text {a }}$ oben verstlimmelung zeigt, während auf bl. $6^{\text {b }}$ oben ein stluck von der glosse und an der seite die anfänge der zeilen des textes und die diesen entsprechenden stellen des holzschnittes auf bl. $6^{\text {a }}$ fehlen. Die bll. 1 und 3 sowie 4 und 6 werden beim beschneiden übereinander gelegen haben.

Für das $D$ in v. 1 und das $G$ in v. 90 ist, wie es auch der abdruck zeigt, auf 2 zeilen raum gelassen; das (sog. lombardische) D in v. 146 ist zwar grösser als die ubrigen anfangsbuchstaben, doch erstreckt es sich nicht bis in die folgende zeile hinunter. Etwa 1 zeile leeren raumes befindet sich zwischen v. 89, v. 109, v. 160, v. 222 und der jedesmaligen folgenden tiberschrift, ferner nach der capiteluberschrift auf bl. $5^{\mathrm{b}}$. Auf bl. $7^{\mathrm{b}}$ ist der rest der seite nach der uberschrift zu cap. 24 unbedruckt, wol deshalb, weil der folgende holzschnitt, die ganze seite einnehmend, hier keinen platz mehr hatte. Die voll ausgedruckte seite enthält 29 (wie bl. $5^{a}, 7^{a}$ ) od. 30 zeilen (wie bl. $2^{a}$ ). ij und $y$ sind unterschieden. Druckfehler finden sich an folgenden stellen: v. 6 daer statt dat; v. 14 clockinghen statt cloclinen; v. 55 pranc statt prant; v. 146 an statt ay; v. 173 bescaet statt bestaet; v. 175 tachter statt lachter; v. 217 wederslach statt vederslach. An einer stelle, wo Hoffmann geändert hat, braucht man keinen druckfehler anzunehmen: v. 119: in den heeren rinck, - Hoffnann: in der h. $r$.

Papier, druck und bolzschnitte beweisen, dass der druck aus der officin des G. Leeu in Antwerpen um 1487 hervorgegangen ist; herr oberbibliothekar $\mathrm{H}$. Bradshaw hat, wie er mir freundlichst mitteilen lässt, daran nicht den geringsten zweifel: die typen seien dieselben, mit denen Leeu nach 1486 druckte, während andere umstände zeigten, dass es vor 1488 gedruckt ist.

Den originalblättern, welche 1870 aus der versteigerung der Culemannschen sammlung in London für die universitätsbibliothek in Cambridge erworben wurden, liegt der Culemannsche abdruck bei; einige kurze notizen liber denselben dürften am platze sein, zumal da das Cambridger exemplar 
dieses abdruckes (welches wahrscheinlich, wie aus verschiedenen umstånden hervorgeht, nur der correcturbogen ist) das einzig elerhaltene zu sein scheint; denn ausgedehnte nachforschungen bei herrn senator Culemann, bei andern privaten und bei bibliotheken nach einem exemplar des abdruckes waren erfolglos. Er führt den titel: 'Brokken eens ouden druks van den Reynaert in verzen' und ist so eingerichtet, dass auf der rïckseite eines blattes der text des fragments, auf der gegenuberstehenden stirnseite des folgenden blattes die entsprechenden verse aus Reinke Vos und aus Reinaert (nach Grimm's ausgabe) stehen. Die typen und die holzschnitte sind denen des originals nachgeahmt. Der text ist buchstaben-, zeilen- und seitengetreu abgedruckt, jedoch nicht frei von allerdings geringftigigen druckfehlern; nicht widergegeben sind einige im original durchschnittene buchstaben und folgende zeilen: wert alhier den ghierigen houelinck gheleert dat (= der ersten zeile des fragments), Diet hoorden worden daer by in vare (= v. 17), Niet nt en mochte daar hi inq $\left(=\right.$ v. $\left.42^{\mathrm{b}}\right)$. Endlich fehlen die verse $72-89$ sowie die uberschrift zu dem hierauf folgenden capitel $\left(=\right.$ orig. bl. $3^{\mathrm{n}}$ ), der holzschnitt auf orig. bl. $3^{\text {b }}$ (welcher im orig. mehr beschnitten ist als der gleiche, im abdruck widergegebene, auf orig. bl. $4^{\mathrm{a}}$ ) und der holzschnitt auf orig. bl. 6a.

Ich lasse jetzt den text des bruchsticks folgen; verszahlen aus $R$. II und $R$. V. sowie die bezifferung der verse des bruchstucks, beschreibung der holzschnitte und angabe der bll. sind von mir hinzugefugt.

wert alhier den ghlerighen houelinck gheleert dat hij soe rele niet rapen en sal dat hi mids dien niet en come in soedanighen gate daer hij niet weder wt comen en kan twelck alhier oeck byden wolf beteykent wert want hij sinen buyck soe vol ghe gheten hadde dat hij niet weder wt den gate ghe comen en konde aldaer hij in ghecropen was. Hier wert oeck ghethoent dat die schalcken bedrieghen heeren ende vrouwen.

(R. II 1513-1588) ie coninck en is mij niet ontgaen

(R. V. 1413-1492) Ic hebbe hem dicke scande ghedaen 
Ende sine wine der coninghinnen

Dat si spade sal verwinnen

(5) Sij sijn ghescandalizeert by mij

Noch hebbe ic daer segghic di

Ysengrine mee: bedroghen

Dan ic soude segghen moghen

Dat icken oom hiet was beraet

(10) Ysengrine die mi niet bestaet

Ic maecten monick ter elmaren

Daer wij beyde beghenen waren

Dat hem zeere wort te pinen

Ic deden in die clockinghen

(15) Binden beyde sine voete

Dat luden dochte hem sijn soe soete

(bl. $\left.1^{b}\right)$

Diet hoorden worden daer by in vare

Ende waenden dattet die duuel ware

Sij liepen daer sij tluden hoorden

(20) Ende eer hi conste in corten woerden

Ghesegghen ic wil mij begheuen

Was hem wel na ghenomen tleuen

Ic dede hem of barnen thaer

Soe na den vel dat wel naer

(25) Die zwaerde hem inden liue cramp

Sint leerde icken dat was sijn ramp

Visschen vanghen op eenen dach

Daer hi ontfinck menighen slach

Oec leyde icken tot spapen van bloys

(30) In al dat lant van vermendoys

En woende gheen pape rijker

Dese pape had een spijker

Daer menich goet vet baeck in lach

Daer hi ontfinck menighen slach

(35) Anden spijker had hi een gat

Ghemaect ende in dat

Dede ic ysegrine crupen

Daer hi runtvleysch vant in cupen

Ende vetter baken alsoe vele

(40) Dies liet hi gaen doer'sijnkele

Soe groten hoop bouen maten

Dat hi wten selnen gaten

(bl. 2a) (42b) Niet wt en mochte daar hi inq...

Dat hem sinen grooten buyck benam 
Doe moeste hi claghen sulck ghewin

(45) Want daer hi hongherich quam in

En mocht hi sat niet comen wt

Ic ghinck ende maecte groot gheluut

In dat dorp ende groot gherochte

$\mathrm{Nu}$ hoert hoe ict daer toe brochte

(50) Ic liep daer die pape sat

Ouer tafel ende at

Ende voer hem stont een capoen

Dat was een dat beste hoen

Datmen wiste in eenich lant

(55) Dat hoen ic mitter vaert pranc

Ende liep hene daer ic mochte

Doe maecte die pape groot gherochte

Ende riep lude vanc ende slach

Ic waen nye man dat wonder en sach

(60) Dat mij een vos rooft mijn hoenre

In mijn huys wie sach yecoenre

Dief ende daer ic sie toe

Sijn tafelmes greep hi doe

Ende warp na mij mer ic ontvoer

(65) Dat mes bleef steken inden vloer

Hij stack die tafel datse vloech

Ende volchde mij mit stemmen hoech

Roepende slach ende va

Ic vaste voren ende hi na

(ii) En mit hemlnyden een groot ghetal

Die mijn quaetste meenden al hiij

(bl. 2b) Holzschnitt, die ganze seite einnehmend; facsimile desselben bei Hoffinann.

(bl. $3^{\text {a) }}$

(R. II, 1637-1654) Doen sprack reynaert wij sijn verm....

(R. V. $1556-15 i 6)$ Uf this boerte $d$. . ghi mij vertelt

Want wat ic soecke ic en vinde niet

(i5) Ic sprack oom wats $v$ gheschiet Cruypt een luttel noch bat in

Men moet wel pinen om ghewin

Ic hebse wech diere voren saten

Dus croop hi in bouen maten

(80) Dat hi die hoenren te verre sochte

Ic sach dat icken honen mochte

Ende stacken dat hi ouer voer 
Ende quam gheuallen opten vloer

Want die haenbalcke was smal

(85) Ende gaf eenen groten val

Dat si ontspronghen alle dyer sliep...

Die daer byden viere laghen si riep..

Datter doer dat valdore gat

Gheuallen ware si en wisten wat

If Hoe dat reynaert sijn biecht is . . . . . . .

gende ende slutende: ende hoe hij daer . . . .

baert te houe weert ghinck ende . . . . . .

inden weghe ghebuerde $\mathrm{Da}$. . . . . . . .

(bl. 3v) Ein die ganze seite einnehmender holzschnitt; vor einem kloster, welches im hintergrunde sichtbar ist, steht links vom zuschauer das wirtschaftsgebaude, in dessen nähe sich hühner und ganse aufhalten; rechts schreitct Reinke mìt nach den hühnern zurückgekehrtem kopfe, während Grimbart sich mit aufgehobener vorderpfote warnend Reinke zunendet.

(bl. $\left.4^{a}\right)$ Der holzschnitt von bl. $3^{b}$ widerholt.

(R. II, 1751-1770) rimbaert sach wel dit ghelaet

(R. V. 1665-1684) Ende seyde vuyle onreyne viaet

Hoe laetty uwe ooghen omme gaen

Reynaert sprack neue dats misdaen

Dat ghi mit uwe verlopende woort

(95) Mij wt mijn ghebede dus stoort

Laet mij doch lesen een Pater noster

Der hoenre zielen vanden clooster

Ende den gansen te ghenaden

Die ic dicke hebbe verraden

(100) Doe icse dese heylighe nonnen

Mit mijnre list heb of ghewonnen

Grimbaert balch hem mer reynaert

Had ymmer thooft ten hoeure waert

Tot si quamen ter rechter straten

(105) Die si te voren hadden ghelaten

Daer keerden si te houe waert

Och hoe seere beuede reynaert

Doe hij den houe began te naken

Daer hi seer in meende misraken

I Hoe reynaert coemt in presencie van den coninc die welke hi obedientelick toeniget ende vindet daer elkerlijck ouer hem claghende Dat. xxij. capittel 
(bl. 5a) (119) Nochtans dede hi als die onuervaerde

(R. II, 1778-1806) Ende liet hem bat dan hem was

(R. V. 1692-1722) Hi ghinck mit sinen neue den das

Cierliken doer die hoochste strate

Alsoe moedich van ghelate

(115) Als of hi sconincs sone waer

Ende hi oec van enen haer

Jeghen nyemant en hadde misdaen

Voer nobel den coninck ghinck hi staen

Midden inden heeren rinck

(120) Ende seyde god die alle dinck

Gheboot die gheue $\mathbf{v}$ coninck heer

Langhe blijscap ende groot eer

Ic gruet $\mathbf{v}$ heer ic hebbe recht

'Ten hadde nye coninck enen knecht

(125) Soe ghetrouwe ieghen hem

Als ic $\nabla$ ye was ende noch ben

Dat ic oeck dicke bin werden anschijn

Nochtan sulcke die hier sijn

Souden mij gheerne $v$ hulde rouen

(130) Mit loghen woudijs hem ghelouen

Mer neen ghi niet god moets $\nabla$ lonen

Het en betaemt niet der cronen

Dat ghi den schalcken ende den fellen

Te licht ghelouet van dat si tellen

(135) Nochtan wil icx gode claghen

Daer isser te vele in onsen daghen

Die mitter loosheyt die sij konnen

Die vorderhant $n \nabla$ hebben ghewonnen

(bl. $\left.5^{b}\right) \quad O u e r$ al in heren houen

(140) Dat sij soe verre comen bouen

Die schalke sijn in dien gheboren

Dat sij den goeden beraden toren

Dat wreke god an haer leuen

Ende moet hem sulck loongheuen

(145) Als sij van rechte wel sijn waert

Die coninck sprack an reynaert

Onreyne vuyle lose drunt

Hoe wel coendy uwen saluut

Maer ten baet $\mathbf{v}$ niet een kaf

(150) Coemt uwes smeckens af

Ic en worde by smecken niet $v$ vrient

Dat ghi mij dicke wel hebt ghedient

Dat wort $\mathrm{v}$ nv te rechte ghegouden

Ghi hebt oec wel den vrede ghehouden

(155) Dien ic gheboot ende hebbe ghesworen 
Owij wat heb ic al verloren

Sprack cantecleer aldaer hij stont

Die coninck sprack hout uwen mont

Her cantecleer ende laet mij spreken

(160) Ic moet antwoerden sine treken

П Hoe dat die coninck reynaert zeere confu selijck ende wredelijck toe spreect om der groo ter quade felle daden daer hij of beclaecht is ende hoe dat hem reynaert weder verantwoert soe hi best kan. Dat. xxiij. capittel

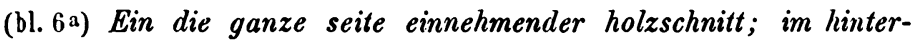
grundc links auf einer anhöhe kniet Reinke demütig vor dem dachs. Im vordergrunde links der wolf, der hahn und der kater liegend; rechts sieht man noch den kopf des baren und den schwanz des lowen.

(bl. $6^{\mathrm{b}}$ ?

e nichte vanden claghers voerder be

. . . . . . wijl gheuanghen.

(R. II, 1829-1852) . . . . ef sprack hij fel reynaert

(R. V.1723-1790) . . . . i mij lief hebt ende waert

. . . den lachter mijn

(165) . . . . . bert ende brune

. . . loedich is sijn crune

. . . . et vele schelden

. . . e $\nabla$ kele salt ontghelden

(170) . . . . . er xpriste fijle

. . . naert here ende of brune

. . . edich heeft die crune

. . . wat bescaet mij dat

. . . yts honich at

(175) . . . die dorper tachter dede

. . . brune soe starcke lede

. . . ghen of versproken

. . . et hi hadt ghewroken

. . . . in dat water

(180) . . . e tybaert die kater

. . . . chde ende wel ontfinck

. . . stelen ghinck

. . . hays sonder minen raet

. . . die pape dede quaet 
(bl. 7a)

(185) Bylode soude ic des ontghelden

Soe mostick mijn gheluc wel schelden

Niet daer by her coninck lyon

Wat ghi wilt dat moechdy doen

Ende ghebieden ouer mij

(190) Hoe goet hoeclaer mijn sake sij

Ghi moecht mij vromen ende scaden

Wildy mij sieden ofte braden

Ofte hanghen ofte blenden

Ic en mach $v$ niet ontwenden

(195) Wij sijn alle in uwen bedwanck

Ghi sijt starck ende ic bin cranck

Mijn hulp is cleyn die uwe is groot

Voerwaer al sloechdi mij doot

Dat waer $v$ eene crancke wrake

(200) Recht in deser seluer sprake

Spranck op bellijn den ram

Ende sijn moeye die mit hem quam

Dat was dame olewij

Bellijn sprack nv toe gaen wij

(205) Alle voert mit onser claghen

Brune spranck op mit sinen maghen

Ende tybert sijn gheselle

Ende ysegrim die snelle

Die haze ende dat euerzwijn

(210) Elck wilde in die claghe sijn

Panthel die kemel ende bruneel

Die gans dat wezel ende tlampreel

Boudwin den ezel borreel den stier

(bl. ib) Dat hermel die wesel waren oeck hier

(215) Cantecler ende sijn kinder

Claechden seer haren hinder

Ende maecten groot wederslach

- Dat troeseel eencleen beiach

Liep oeck mede in deser scare

(220) Alle dese ghinghen openbare

Voer haren heere den coninck staen

Ende deden den vos reynaert vaen

9 Hoe die coninck te recht sittet ende gheeft

die sentencie datmen reynaert vanghen sou

de ende byder kelen hanghen Dat. xxiiij. capittel

Der nachweis der urspringlichen lage der blätter, welchen wir im folgenden versuchen wollen, ist um deswillen nötig, weil er uns eine sichere grundlage gewähren kaun zur weitern 
vergleichung der liberschriften und der glosse in $r$ und h. Fur denselben haben wir nehrere anhaltspunkte: einmal die tatsache, dass d durchgängig mit den verszahlen in $b$ ubereinstimmt, dann die signatur hiij unten auf bl. $2^{a}$, ferner dass entweder 29 (wie auf bl. $5^{\text {" oder }} 7^{\text {a }}$ ) oder 30 zeilen (wie auf bl. $2^{a}$ ) auf der vollbedruckten seite stehen, sowie endlich die beobachtung, dass meist auf die capiteliberschrift ein holzschnitt, dann eine titelglosse, endlich der text folgte (vgl. cap. 19 (?) nach v. 89 ; besonders cap. 23 nach v. 160 , auf welche tuberschrift auf

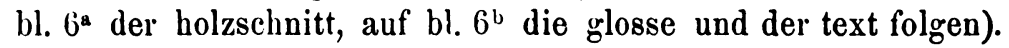
Aus der signatur hiij geht hervor, dass das erste und daher auch das letzte blatt des bogens $h$ verloren gegangen ist; ferner ist nicht erhalten bl. 4 des bogens h, das mit dem erhaltenen bl. 2 des fragments ein doppelblatt bildete. Es bestand demnach der bogen h aus 6 bll. 1) Auf der rickseite des ersten blattes des bogens $h$ wird ein holzschnitt gestanden haben, während unten auf der stirnseite desselben blattes die überschrift zu dem capitel (dem 17.?), dessen text auf bl. h 2 $=$ bl. 1 des fragments gerettet ist, sowie einige verse des vorhergehenden (16.?) capitels (vielleicht $=$ R. II, 1490-1512) standen. - Auf das bl. h 4, welches mit dem bl. 2 des fragments ein doppelblatt bildete, müssen die verse R. II, 1589—1636 $=48$ verse verteilt werden; gleichmässige verteilung von je 24 versen auf stirn- und rückseite oder die anordnung: v. 15891617 auf der stirn-, v. 1618-1636 auf der rückseite wird nicht angenommen werden dürfen, $d a$ in beiden fällen zuviel raum verschwendet sein wurde. Aehnliches kommt zwar vor auf fragm. bl. $7^{\text {b }}$ wo jedoch der leere raum gerechtfertigt ist, da fur den folgenden holzschnitt, der die ganze seite einnahm, nicht platz genuz vorhanden war und im letztern falle der zusanumenhang der erzïlilung auch fortlaufenden druck forderte. Auf den richtigen weg kann uns h fuhren; dieses setzt auf s. 38 in der beichte Reinaerts bei der erzählung von weitern gefahren, in welche Isegrim durch Reinaert gebracht sei, ein neues (das 18.) capitel an, (whihrend $r$ diese einteilung nicht vornimmt): der beginn des capitels entspricht $r, 1534$; R. II, 1618. Dem entsprechend möchte ich vermuten, dass unser

1) Vgl. die iibersicht unten s. 20.

Beitrüge zur geschichte der deutschen sprache. VHI. 
bruchstück ebenfalls vor dem R. II, 1618 entsprechenden verse eine capiteluberschrift gehabt habe. Diese nun aber alleïn (naturlich zusammen mit jenen 48 versen) kann der verlangten ausfüllung des bl. h 4 nicht genügen, da keine der capitelüberrschriften des fragm. mehr als 5 zeilen einnimmt (frag. bl. $3^{\mathrm{a}}=$ 4 zeilen; bl. $4^{\mathrm{b}}=3$ zeilen; bl. $5^{\mathrm{b}}=5$ zeilen; bl. $7^{\mathrm{b}}=3$ zeilen $)$. Es werden daher wol noch einige zeilen glosse nach der capiteluberschrift gestanden haben, die dann dem inhalt, vielleicht auch der form nach R. V. Glosse z. I, 17, s. 56, $10 \mathrm{ff}$. entsprechen wiirden. Hiernach mögen auf bl. h $4^{\mathrm{a}}$ gestanden haben: vv. = R. II, 1589-1617, auf bl. h $4^{\text {b }}$ überschrift und glosse (zu cap. 18 ?) und rom text $\mathrm{vv} .=$ R. II, 1618-1636.

Die folgenden fehlenden blätter müssen zusammen einer erörterung unterzogen werden. Es ist das letzte bl. des bogens $\mathrm{h}$ und das erste bl. des bogens $\mathrm{i}$, welch letzteres, wie noch aus dem am bl. 7 des fragments erhaltenen rande ersichtlich ist, mit diesem ein doppelblatt ausmachte. Auf fragm. bl. $4^{\text {b }}$ sind oben 4 zeilen abgeschnitten: vers 90 des fragm. ist gleich R. II, 1751 und vers 89 des fragm. entspricht R. II, 1654. Es sind demnach 96 verse $(=\mathrm{R}$. II, 1655-1750) zu verteilen auf bl. h 6 und auf bl.i 1. Eine einfache durchteilung, so dass etwa 24 verse auf jede seite kämen, ist hier ebensowenig am platze wie oben. Bot sich uns bei der zuletzt besprochenen stelle $h$ als controle zur bestimmung einer capiteleinteilung, so haben wir hier durch das hinzukommen von $r$ eine um so grössere stutze: an der R. II, 1713 entsprechenden stelle beginnt $r$ sowol ein neues (das 18.) capitel, als auch h (das 20.), wodurch eine capiteleinteilung an dieser stelle auch des fragments höchst wahrscheinlich anzusetzen ist. Aus andern grtinden wird man zu der annahme gefuhrt, dass $d$ mit v. 90 ein neues capitel anfieng, welches sich weder in $r$ noch in $\mathrm{h}$ findet $(\mathrm{d}, 90=\mathrm{l}$, $1665=$ h, s. 40,22$)$ : der für ein grosses $G$ freigelassene platz in $d, 90$ scheint darauf hinzuweisen (ebenso für das $D$ in $d, 1$ ), vor allem aber der holzschnitt auf fragm. bl. $4^{\text {a }}$, so dass wir nach diesem auf fragm. bl. $4^{\mathrm{b}}$ oben (dem weggeschnittenen teil) eine glosse anzusetzen genötigt werden. Demgemäss stand die $\mathrm{zu}$ dieser glosse und dem folgenden text gehörige capiteluberschrift unten auf bl. i $1^{\mathrm{b}}$ : der alte druck hat eine vorliebe dafür, die capitelüberschriften unten an das ende einer seite 
zu setzen, $\mathbf{v g l}$. die betreffenden tiberschriften auf bl. $3^{\mathrm{a}}, 4^{\mathrm{b}}, 5^{\mathrm{b}}$, $7^{b}$ des fragments. Nehmen wir nun an, dass diese capiteluberschrift etwa 4 zeilen einnahm, so mag der ubrige teil des bl. $i 1^{\text {b }}$ die verse enthalten haben, welche R. II, 1726-1750 entsprechen. - Nun zeigen uns aber, wie oben bemerkt, $h$ und $r$, dass noch eine zweite capiteleinteilung auf einem dieser beiden blätter vorgenommen ist; rechnen wir von 1725 aufwärts bis zum abschnitt $=$ R. II, $1713(=\mathrm{r}, 1627$; h, cap. 20), so wird auf bl. i $1^{\text {a }}$ vor den R. II, 1713-1725 entsprechenden versen eine glosse und davor eine capitelüberschrift gestanden haben: ein holzschnitt $\mathrm{zu}$ diesem capitel wird nicht geliefert worden sein, weil ein solcher, die ganze seite einnehmend, sich nicht in die 96 verse umfassenden 2 bll. einfügen lässt. Jetzt haben wir noch auf bl. $\mathrm{h} 6$ und auf einen teil des bl. i $1^{\text {a }} \mathrm{zu}$ verteilen eine glosse $\mathrm{zu}$ der auf bl. $3^{\mathrm{a}}$ des fragments erhaltenen uberschrift sowie die vv. = R. II, 1655-1712, deren anordnung möglicherweise diese ist: bl. $\mathrm{h} 6^{\mathrm{a}}$ enthielt die glosse $\mathrm{zu}$ der uberschrift auf fragm. bl. $3^{\mathrm{a}}$ nebst den vv. = R. II, 1655-1676, bl. $\mathrm{h}^{\mathrm{b}}$ die $\mathrm{vv}$. $=$ R. II, 1677-1706, bl. i $1^{\mathrm{a}} \mathrm{vv}$. $=$ R. II, 1707-1712, sowie überschrift und glosse zu capitel 20 (?) nebst den vv. $=$ R. II, 1713--1724.

Diese herstellung hat gegen sich einmal das fehlen einer capiteleinteilung in $r$ (sie musste stehen $r, 1665$ ) und in $h$ (musste stehen $h, 40,22$ ) und dann den umstand, dass das angesetzte capitel nur eine verhältnismässig kleine anzahl von versen (d, 90-109) enthalten wiirde. Um des willen könnte man folgendermassen anordnen: bl. h $6^{\text {? }}$ glosse zur capitelüberschrift auf bl. $3^{\mathrm{a}}$ des fragments und vv. $=$ R. II, 1655-1676; bl. h $6^{\mathrm{b}} \mathrm{vv}$. $=$ R. II, 1677-1705; bl. i $1^{\mathrm{a}} \mathrm{vv}$. $=$ R. II, 1706-1712, uberschrift und glosse zu einem neuen capitel nebst $\mathbf{v v . ~}=\mathbf{R}$. II, 1713-1717; bl. i $1^{\mathrm{b}} \mathrm{vv}^{\mathrm{v}}=\mathrm{R}$. II, 1718-1746; bl. i $2^{\mathrm{b}}$ oben vv. $=$ R. II, 1747-1750 (gleich dem weggeschnittnen teil). Doch da wir auch hierbei ebensowenig einen holzschnitt gewinnen können für das mit v. 1713 beginnende capitel, als bei der obigen anordnung, und wir mit letzterer dem streben des alten drucks, die capitelüberschriften möglichst an das ende einer seite, dann einen holzschnitt, endlich die glosse zu setzen, entgegenkommen, so möchte ihr der vorzug zu geben sein: $r$ und $h$ können zusammengezogen haben. 
Es ertabrigt noch, das bl. i 3 einzureihen, von dem noceh der rand an fragm. bl. 5 erhalten ist. Bl. $4^{\text {b }}$ des fragmenits endigt im text mit v. $109=\mathrm{R}$. II, 1770, woran sich die ubeerschrift zu cap. 22 schliesst; bl. $5^{\text {a }}$ des fragments beginnt mit v. $110=\mathrm{R}$. II, 1778; wir haben demnach auf bl. i 3 nur 7 verse text $\mathrm{zu}$ verteilen, das librige wird von einem hollzschnitt und einer glosse eingenommen gewesen sein; demzufolgge stellen wir her: bl. i $3^{\mathrm{a}}$ holzschnitt; bl. i $3^{\mathrm{b}}$ glosse zu capittel 22 und vv. $=$ R. II, 1771-1777.

Die gewonnene herstellung fassen wir noch einmal iin übersichtlicher darstellung zusammen.

\section{Bogen h.}

bl. 1a ... fehlt [? vv. = R. I1, 1490-1512; überschrift zu cap. 17 (??). vgl. R.V. I, 17; h cap. 17.]

bl. $1^{\mathrm{b}}$... fehlt [? holzschnitt.]

bl. $2^{\mathrm{a}}=$ bl. 1a $^{\mathrm{a}} 4$ zeilen der glosse abgeschnilten] glosse zu cap. 17 ((?) (vgl. R. V. gl. z. I. 17, s. 54, 8-19; h, gl. z. cap. 11i, s. 38,21$)$ und vv. $1-16=$ R. II, $1513-1528$.

bl. $2^{\mathrm{b}}=\mathrm{bl} .1^{\mathrm{b}}[v v .=R . I 1,1529-1532$ abgeschnitten $] \mathrm{vv} .17-42=$ R. II, 1533-1558.

bl. $3^{\mathrm{a}}=$ bl. $2 \mathrm{a}$ vv. $42^{\mathrm{b}}-71=$ R. II, $1559-1589$.

bl. $3^{b}=$ bl. $2 b$ holzschnitt.

bl: $4^{a}$... fehlt [? $\left.v v .=R . I I, 1589-1617\right]$.

bl. 4 b ... fehlt [? überschrift (vgl. R. V. 1534; h, cap. 18, s. 38) umd glosse (vgl. R. V.gl. z. I, 17, s. 56, $10 \mathrm{ff}$.; h fehlt) $z$ u cap. 18 (?) und $v v .=R .11,1618-1632]$.

bl. $5^{\mathrm{a}}=$ bl. $3^{\mathrm{a}}[v v .=R .11,1633-1636$ abgeschnitten $]$ vv. $72-59=$ R. II, 1637-1654; überschrift (vgl. R.V. 1577; h, cap. 119) zu cap. 19 (?).

bl. $5^{b}=$ bl. $3^{b}$ holzschnitt.

bl. ba ... fehlt [? glosse (vgl. R. V. gl. zu I, 17, s. 56, 18 ff.; h fehlt) zu cap. 19 (?) und $v v .=R .11,1655-1676]$.

bl. $6^{b}$... fehll [? vv. =R. 11, 1677-1706].

Bogen i.

bl.1a') .. fehlt [? vv. = R. II, 1707-1712; überschrift (vgl. R. V. I, 18; h, cap. 20) und glosse (vgl. R. V. gl. z. I, 18, s. 58; $h$ fehlt) zu cap. 20 (?) und vv. R. II, 1713-1725].

1) Oder bl. 1a $\ldots$ fehlt $[v v .=R . I I, 1707-1712 ;$ überschrift umd glosse zu einem neuen cap. und $v v .=R . I 1$, 1713-1717]. 
b. 1 b ... fehlt [? rv. $=$ R. II, 1726-1750; überschrift zu cap. 21 (?) (R. V. fehlt, vgl. $1665 ;$ h felllt, vgl. s. 40, 22)].

bl. $2^{\mathrm{a}}=$ bl. $4^{\mathrm{a}}$ holzschnitt.

bl. $2^{b}=$ bl. $4^{\text {b }}$ [weggeschnitten 4 zeilen glosse zu cap. 21 (?) vgl. $R$. V. gl. z. I, 18 s. $58,14 \mathrm{ff}$; $h$ fehlt] vv. $90-109=\mathrm{R}$. II, $1751-1770$; iberṣchrift (vgl.r, I, 19; h, cap. 21) zu cap. 22.

bl. 3a ... fehlt [? holzschnitt].

bl. $3^{\text {b } . . . ~ f e h l t ~[? ~ g l o s s e ~(v g l . ~ R . ~ V . ~ g l . ~ z . ~ 1, ~ 20, ~ s . ~ 63 ; ~ h, ~ g l . ~ z u ~ c a p . ~ 21) ~}$ zu cap. 22 und $v v .=R .11,1771-1777]$.

bl. $4^{\mathrm{a}}=$ bl. $5^{\mathrm{a}}$ vv. $110-138=$ R. II, 177s-1806.

bl. $4^{\mathrm{b}}=$ bl. $5^{\mathrm{b}}$ vv. $139-160=\mathrm{R}$. II, $1807-1828$ und iiberschrift (vgl. R. V. $1723 ; h, s .42,6)$ zu cap. 23.

bl. $5^{\mathrm{a}}=$ bl. $6^{\text {a }}$ holzschnitt.

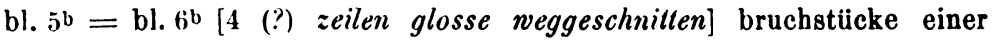
glosse (vgl. R. V. gl. zu I, 20, s. 63; h fehlt) zu cap. 23 und vv. $161-184=$ R. II, $1829-1852$.

bl. $6^{n}=$ bl. in vv. $185-213=$ R. II, 1853-1881.

bl. $6^{\mathrm{b}}=\mathrm{bl} .7^{\mathrm{b}}$ vv. $214-222=\mathrm{R}$. II, $1882-1890$ und iiberschrift (vgl. R. V. I, 20; h, cap. 23) zu cap. 24.

\section{Die äussere einteilung.}

1. Die einteilung in 4 part.

Von den uns erbaltenen $\mathrm{nl}$. gedichten und prosa-auszigen der Reinbarts-sage kennt kein einziges die einteilung in 4 bucher, wie sie im R. V. vorgenommen ist. Es ist daher bei dem glucklichen zufall, der uns das bruchstuck d erhalten hat, nicht genug zu bedauern, dass nicht aus einem spätern teile des gedichts blätter gerettet worden sind (unsere fragmente sind gleichstehend mit capp. 17--20 des ersten buches des R. V.) - wir würden dann eine absolut sichere beantwortung der frage geben können, ob die vorliegenden bruchstucke dem Hiurek van Alckmer zuzuweisen sind oder nicht, denn dieser hatte seine bearbeitung in vier teile geteilt, wie uns die erste vorrede zu R. V. (Lubben s. IV, 6) berichtet. An der richtigkeit dieser angabe ist nicht zu zweifeln, da die ganze erste vorrede wörtlich aus dem nl. übernommen zu sein scheint hierfür als beweis können, abgesehen von andern grunden, die worte gelten de sêr genôchlik is to lesen (Lübben III, 18),

bl. $1^{\mathrm{b}} \ldots$ fehll $[v v .=R . I I, 1718-1746]$.

bl. $2^{\mathrm{a}}=$ bl. $4^{\mathrm{a}}$ holzschnitt.

bl. $2^{\mathrm{b}}=$ bl. $4^{\mathrm{b}}[v v .=R .1 I, 1747-1750$ weggeschnitten $]$ u. s. w. 
die auf dem titelblatt von $\mathrm{h}$ (und aller folgenden volksblicher) wiederkehren: een seer ghenuechliigke ende vermakeliicke historie (vgl. auch $\mathrm{p}$, bl. I ende voer den verstandelen seer ghenuechtelijck ende oeck profitelijk). 'Die angabe, dass es ût walscher' unde franzosescher sprake gesocht is unde ummegesat in dudesche sprake (Lübben IV, 3) ist ungenau und blieb vielleicht nur stehen, weil sie sich im eingange von R. I, 8.9 befindet' (Grimm, R. F. s. CLXXVI); des glossator zu R. V. I, 3, s. 9, 17 nabm diese versicherung H's v. A. freilich für baare münze indem er sagt: in Lomberdien und Wallant, dar dit bôk ersten gedichtet is. Die vierteilung des gedichts ist also nicht im nd. zuerst eingefuhrt, was auch ausserdem noch bestätigt wird durch die worte des glossators zu I, 39 (Lubben s. 109, 12) de lerer . . . beslut dar mit dat erste bôk.

Ueber diese vierteilung soll nun nach der uberschrift (die vielleicht ursprünglich randbemerkung zum voraufgebenden war) zur anderen vorrede: Wo dit bôk wert gedelet in IIII part in dieser gehandelt werden; das geschieht jedoch nicht, man findet auch nicht den leisesten ansatz zu einer begrlindung der einteilung in 4 teile, vielmehr erfahren wir nur, dass es in der menschlichen gesellschaft vier stände gibt, durch deren nähere charakterisierung der glossator dem leser ein besseres verständnis des gedichtes beibringen zu können glaubt (Lübben IV, 9. 10). J. Grimm, R. F. s. CLXXVII möchte diese vorrede dem Nicolaus Baumann zuschreiben (während er für die erste Henrik v. Alckmer als verfasser annimmt): er musste zu dieser ansicht kommen, nachdem er s. CLXXII die worte de froien siripen, de so westwart werden genomet (Lubben II, 29) nur verstehen $\mathrm{zu}$ können erklärt, wenn man annimmt ${ }_{n}$ dass der verfasser, jetzt ostwärts wohnend, seiner angeborenen (?) westlichen, d. h. westphälischen mundart eingedenk blieb ${ }^{\star}$. Es scheint Grimm entgangen zu sein, dass schon in der Goudaer ${ }^{1}$ ) prosa in der

1) Nach Hoffmann Hor. Belg. XII, 5 anm.* ist die Delfter prosa von 1485 ein nachdruck der Goudaer von 1479; was mir herr oberbibliothekar dr. Campbell gütigst bestätigte und ferner mitteilte, dass eine vergleichung beider drucke lehrt, dass der Delfter nachdruck vom anfang bis fol. 69 mit dem Goudaer druck seite für seite, zeile für zeile stimmt; von fol. 70 an hat der Delfter 2 oder 3 zeilen mehr, dann und wann stimmen auch die zeilen nicht mehr genau, was aber gegen ende wider 
vorrede I. I" diese vierteilung, wenn auch nicht als solche direrst gokennzeichnet, so doch angedeutet ist; es heisst dort nämlich ,.. onder den raet daer herē eñ p̄latē gheestelic ende waerlic $\bar{n}$ vnder die coopludē. eñ nec onder den gemeenē volc. ${ }^{\star}$ - Seitdım nun aber das von Martin in Freiburg aufgefundene exemplaı des nl. volksbuchs von 1564 vorliegt, wissen wir mit bestimmtieit, dass diese begründung der vierteilung in der zwoiten vorrede, wenigstens in ihrem grundstock, nicht erst vom ubasetzer des R. V. herrührt. Das volksbuch führt uns dieselbe zweimal getrennt vor, in dem es zuerst $(\mathrm{h}, 5,6-5,14)$ die vershiedenen kategorien der staeten aufzählt, wobei jedoch der dritto und vierte stuet nicht als solcher bezeichnet, sondern zwei moralische lehren aus dem inhalte des buches vorweg genommen werlen und dann ( $h, 5,20-6,6)$ auseinandersetzt, welcher staet mit den einzelnen tieren gemeint sei. Genau dasselbe, wie aus der folgenden vergleichung hervorgeht, findet sich im R. V., nur mit dem unterschiede, dass in diesem gleich bei jedem stande beigeftigt wird, welche tiere denselben repräsentieren, und dass eine andere reihenfolge eingehalten wird.

1. $(r, I V, 11) \ldots$ stât van den arbeiders ....

$(I V, 19)$ unde bi dessem state so gelikent de meister in dessem boke de arbeidenden deren alse perde, mulen, esels, ossen unde der geliken ....

\section{h}

$(h, 5,7)$ Ten tweeden den staet van den gemeynen volcke....

$(6,3)$ Ende ten lesten sult ghijer oock vinden den staet van den arbeyders, de welcke geleken worden by den arbeydende dieren, alse peerden, Ossen, Ezels, ende dier ghelijcke.

der fall ist. Dio orthographie ist in kleinigkeiten verschieden; druckfehler sind: 1485 fol. $m$ III a half: 1479 alt; 1485 fol. m VIIIb Alaerde: 1479 Blaerde (vgl. Martin, Volksb. s. X); 1485 fol. XXXIX a bende: 1479 ende. - Exemplare des Delfter nachdrucks sind vorhanden; 1. Kgl. bibl. z. Kopenhagen; 2. stadt-bibl. z. Liibek; 3. Bodleian library z. Oxford; 4. senator Vergauwen in Gent; 5. † prof. Serrure in Gent. Vgl. übrigens Campbell, Annales de la typographie Néerlandaise au XV e siècle, La Haye, 1874 no. 976. 977 .

Vom Goudaer Reinaert-druck, 1479, existieren folgende exemplare: 1. Kgl. bibl. im Haag; 2. brit. mus. in London, vgl. catalog. biblioth. Grenvill. p. 601 a. Ein drittes exemplar, welches, wie mir herr oberbibliothekar dr. Campbell berichtet, früher im besitz von Van Damme gewesen ist, scheint verschollen zu sein. Uebrigens hat Buddingh keinen neudruck dieser Goudaer prosa veranstaltet (vgl. Grimm R. F. s. CLXIV). 
2. $(r, I V, 22) \stackrel{r}{r}$ borgerie unde kôplude ...

(IV, 24) $\mathrm{Bi}$ dessen gelikent de meister de deren, dede leven ... alse dat ekerken, de hampster, hasen, kaninen ....

3. (r, IV, 32) de geistliken ...

(IV, 33) Dessen gelikent desse meister bi deme grevinge...

4. $(r, V, 5)$ de vorsten unde heren der werlt, de sik eddel holden ....

$(V, 7)$ Desse gelikent de meister desses bokes bi deme wulve unde bi deme baren. h

(h, 5, 27) [Dander sijn van cleyn. der ende leegher condition ....]

$(6,1)$ ende dese ghelijct den Autheur van desen boecke by den Vosse, Simme, Hont, Cater.

$(h, 5,20)$ in den eersten, de gheestelijcken staet.

$(5,21)$ den gheestelijcken staet wort gheleken by den Dasse.

(h, 5, 6 u. 5, 23) In den eersten hebt ghijer in den staet van den prince ende van sijn bof ... Daer na den staet der Edelen, onder de welcke ...

$(5,26)$ Dese worden gheleken by den Wolf, beyr, Losse ende Luypaert.

Ganz strenge werden bei dieser einteilung im R. V. die tiere doch nicht auseinander gehalten: diejenigen, welche IV, $25 \mathrm{ff}$. den zweiten stand repräsentieren, werden nochmals V, $12 \mathrm{ff}$. bei der unterabteilung der vorsten verwant.

Nach dieser einleitung folgen nun im R. V., indem gleichmässig nit de lerer bewiset $\hat{o} k$ angeknüptt wird, V, 15-37 einige lehren, die man aus der lectüre des buches entnehmen könne, und von denen wenigstens zwei an h anklingen; man vergleiche

$(\mathrm{V}, 25)$ wo de vorsten vaken werden vorleidet vau den logeneren $\hat{t}$ deme wege der rechtverdicheit.

$(V, 29)$ He bewiset ok, dat den vorsten unde heren dat vele nutter is to hebben den wisen in ereme rade, dan den girigen.

\section{$h$}

$(5,9)$ van den luegenaere, hoe ende in wat manieren sy de menschen connen verblinden met hen lueghentale ende schoone woorden.

$(5,15)$ Oock dattet den prince orbaerlijcker is, wijse lieden in sijn hof te hebben, dan ghierige lieden.

Auch kann hierher gezogen werden die ermahnung an den leser, welche wol sicher, wegen der schlussschrift im R. V. s. 226 , schon im nl. stand: 


\section{$r$}

(VI, 23) men den sin der worde, wat de lerer mede menet, schal men merken unde beholden, dar de licht wisheit in. h

$(j, 4)$ Maer leest met verstande ende siet tot wat eynde elck dinck geschreuen is, ghi sulter groote leeringen cnde vnderwijsingen in vinden.

Es steht hiernach fest, dass der verfasser des R. V. auch in der zweiten vorrede nicht selbständig gearbeitet hat: wie weit er sich hinsichtlich der anordnung, der einzelnen ausdrucke u. s. w. an seine rorlage angeschlossen, ist schwer zu bestimmen, jedoch dürfen wir, wenn wir in betracht ziehen, dass das volksbuch doch nur einen auszug aus einem verloren gegangenen nl. exemplar darbietet, wo! zu dem schlusse berechtigt sein, dass der nd. übersetzer, vorzüglich wegen der böchst wahrscheinlichen tatsache des directen ubersetzens der ersten vorrede, auch hier all $\mathrm{zu}$ weites entfernen vom $\mathrm{nl}$. vermied.

Dies gewinnt eine stütze durch die dem nd. fremde construction des verbums gelikenen mit bi (IV, 19, 24, 33; V, 7, 11, 13, 19); dass aber auch die stelle IV, 33-V, 3 in der nl. vorlage schon stand, wird fast zur gewisheit durch die erklärung, welche dem worte greving IV, 33 hinzugefugt wird: de $\hat{o} k$ in etliken landen wert geheten de das und dass die stelle $\mathrm{V}, 1$ doch strafet he se mit vordeckeden worden umme twei sunde, alse umme de giricheit unde unkuscheit fast wörtlich ubereinstimmt mit den worten des volksbuches 5, 22: Ende bedectelijck worden dese begrepen van ghiericheyt ende oncuysheyt.

Nach diesen erörterungen können wir uns an die öfter besprochenen worte IV, 29 de froien stripen, de so westwort werden genumet machen. J. Grimm R. F. s. CLXXII fast dieselben so auf, als ob froie und stripe westfälische ausdrücke seien, durch die der tibersetzer auf seine heimat habe hinweisen wollen. Dem entgegen hat Latendorf im programm des Schweriner gymnasiums 1865 s. 33 behauptet, die erklärung dieser worte wie derjenigen in der iberschrift zu I, 5 sik moiende mit overtogen koggelen so westwert de wise is sei in $\mathrm{nl}$. quellen $\mathrm{zu}$ sucher. Ich schliesse mich des letzteren ansicht deswegen an, weil einmal der ubersetzer (nachdem von Zarncke, Haupts Zeitschr. f. d. Altertum IX, 374 erwiesen ist, dass Baumann, 
der freilich aus Westfalen stammte, nicht der verfasser des R. V. gewesen sein kann) durchaus keinen ersichtlichen grund hatte, westfälische ausdrtucke in den R. V. hineinzubringen und ihm zweitens diese tiernamen und diese sitte, die gestorbenen zu betrauern, sicher fremd waren: denn weshalb begnugte er sich sonst mit dem einfachen zusatze de so nestwort werden genomet und so westwert de wise is, während er im tubrigen doch, wie wir gesehen haben (Lubben IV, 33 s. o. s. 25), das den rechts-elbischen gelïufige wort setzte ohne irgend eine geographische bestimmung und einem unverständlichen worte eine tatsächliche erklärung beifïgt z. b. glosse z. I, 18 s. 58, 11 ypocriserie, dat is schalkheit unde bôsheit to bedecken mit einer gevinseder hillicheit. - Nun kommt jedoch froie weder im nd. noch im nl. vor. Grimm, R. F. s. CLXXII erklärt es $=$ fret, d. h. Frettchen, was lautlich ganz wol möglich wäre; allein dieses ist ein raubtier, das sich nicht mit früchten begnügt. Es ist vielmehr mit herrn prof. Verdam in Amsterdam, nach einer brieflichen mitteilung, die er mir zu veröffentlichen gütigst gestattete, hier einfach ein druckfehler (des Reinke oder seiner vorlage) zu constatieren und zu schreiben: troie. Troie, $=$ truye (sus, scropha; machinae bellicae genus. Kilianus Dufflaeus Etymologicum teutonicae linguae Ultraj. 1623, p. 685) mlat. troja (s. Du Cange s. v.) franz. truie; heute noch existiert das wort im nl. in derselben bedeutung, einige dialecte gebrauchen es fur kaninchen. - Stripe kennen beide sprachen nur in der bedeutung von streifen: es wird an unserer stelle stripe wol ein gestreiftes tier bedeuten, s. Grimm R. F. s. CLXXIII.

Dass der nd. libersetzer die auseinandersetzung uber die tiernamen ${ }^{1}$ ) (Lubben V, 35 - VI, 23) selbständig gearbeitet hat, dafur sprechen die nd. namen; auch darf betont werden, dass s. VI, 3; VI, 22 ausdrïcklich von zuhörern gesprochen wird, die der text ganz ausser acht lüsst, indem er sämmtliche stellen, in denen diese in b angeredet werden, ändert, während die glosse sich zweimal an dieselben wendet (s. 24, 17 und s. 83,6$)$.

Schon oben haben wir gesehen, dass die charakterisierung

1) Die wilde katte Alse (VI, 10) ist zu streichen: vgl. Baethcke, Germ. XIX, 111. 
der 4 stände in dieser zweiten vorrede nichts zu tun hat mit der einteilung des gedichts in 4 teile. Näheren aufschluss, weshalb gerade die vierteilung gewählt worden ist, erbalten wir nicht und dürîen sie auch kaum erwarten, àa kein vernünftiger grund für die zerlegung der fortsetzung in 3 bucher wird angegeben werden können: die natülichste einteilung war die in zwei hälften, von denen die erste 3480 (in r: 3246), die zweite 4314 (in $r: 3598$ ) verse umfasst hätte. Haben wir so keine begründung der vierteilung, so finden wir doch eine glossierung der einzelnen abteilungen in der form einer art vorrede zu dem betreffenden buch, die umfangreicher wird, je weiter wir uns dem ende des ganzen naben: zum ersten buch keine vorrede, zum zweiten nur 5 zeilen, zum dritten nimmt sie schon 10 zeilen ein, und diejenige zum vierten buch, die sich selbst als vorrede bezeichnet, wird so umfangreich wie die erste vorrede zum ganzen werke. Dass dieselbe schon in der bearbeitung des Hinrek van Alckmer gestanden habe, scheint mir zweifelhaft zu sein: in ihrer breiten ausfuhrung eines später noch zweimal [tiberschr. z. IV, 5, s. 203 und glosse z. IV, 5, s. 205] berubrten punktes, mit den hinweisungen auf die bibel und Augustin kann sie nicht fuglich eine korle atlegginge, mit welcher H. v. A. nach der ersten vorrede r, s. IV, 6 seine bearbeitung versah, genannt werden; doch da sie vom nd. ubersetzer erweitert sein, und andrerseits die vorrede zum 3. buch in ibrer kurze sehr wol schon im nl. original gestanden haben kann, wird eine sichere entscheidung nicht möglich sein.

Eine besondere betrachtung verlangt die vorrede zum zweiten buch; in derselben haben wir zwei teile zu unterscheiden: 1. die inhaltsangabe des zweiten buches, 2. die inhaltsangabe der verse $r, 3247-3274$. In den uns bekannten nl. recensionen stehen die genannten verse nicht; sie uberraschen den leser, da sie unverbunden mit dem vorhergehenden auftreten und im folgenden auf sie keinerlei rlicksicht genommen wird. Wenn man so sagen kann, dass sie ohne allen zusammenhang mit den erzählten begebenheiten dastehen, so ist auf der andern seite doch nicht zu leugnen, dass sie sich vollkommen in der situation befinden: Reinkens und Bellins geschlecht ist vom könige für frei erklärt worden; derselbe 
hat durch Lupard die tiere, die vorher am hoftage teilgenommen haben, an seinen hof entbieten lassen, um Isegrim und Brûn volle ehre zu erweisen; aber, sagt die inhaltsangabe, es kamen nicht allein die dere, sondern auch die vögel, die nun vor ihrem erscheinen bei hofe in sieben strophen $z u$ vier versen eine unterredung anstellen, in welcher sie erzählen, dass sie zu hofe entboten worden seien und dass Reinke nicht mehr in des königs gunst stehe; sie beschliessen, Reinke zu verklagen und bedauern nur, sich nicht fruher besprochen zu haben, um Reinke ins verderben zu bringen. Die interpolation ist geschickt gemacht und gibt eine recht hübsche schilderung von dem heldenmut der kleineren vögel, der dem feinde, so lange er noch stark und mächtig war, nicht entgegen $\mathrm{zu}$ treten wagte, jetzt aber, da er weiss, dass sich ihm zu taten keine gelegenheit bieten wird, in wortreicher ausfthrlichkeit zu tage tritt.

Veranlassung zur interpolation scheint man genommen zu haben an dem inhalt des ersten capitels des zweiten buches, in welchem die schilderung der gewalttaten, welche Reinke an der krähe verubt, den grössten platz einnimmt. Nach je vier versen, also nach 3250, $3254^{1}$ ), 3258, 3262, 3266, 3270, 3274 steht ein holzschnitt. Sie weichen in der ausfuhrung ganz und gar von den ubrigen im R. V. ab (sie stellen in blossen umrissen gezeichnete vögelgruppen dar) und sind etwas verkleinerte nachschnitte von holzschnitten, die im Dialogus creaturarum optime moralisatus iucundis fabulis plenus, Gouda, G. Leeu, $1480^{2}$ ), verwandt worden sind, und zwar von folgenden: 1. Dialogus

1) Demnach ist v. 3254 bei Liibben mit Schröder, Reinke-ausgabe, leipzig 1872, die klanmer zu streichen und v. 3255 die lesart des druckes Ja. wy ock des ghelyck vn vnse kynder beizubehalten.

$\left.{ }^{2}\right)$ Und öfters, vgl. Campbell, Annales de la typogr. Néerland. nr. 560 ff. - Mit diesem werke hat $R$. V. nichts gemein, als die angegebenen holzschnitte. Den inhalt des diaiogus bilden 122 in ebensoviel capiteln in lateinischer sprache prosaisch erzăhlte fabeln aus allen drei naturreichen, die auf mir unbekannte quellen zuriickgehen. Oesterley, Romulus, Berlin 1870 erwähnt das werk nicht; bemerkt mag werden, dass der name des Aesop weder in der praefatio noch am schlusse vorkommt (vgl. Oesterley, a. a. o. s. XXIII) und dass das 13. cap. die überschrift hat: de gemmis lapidibns preciosis (vgl. Oesterley, a. a. o. s. XXV). S. auch: Grässe, Literärgeschichte II, 2, 2, s. 714. 1114. 
bl. g $3^{a}$, unter der überschrift: De cigno et coruo dyalogus septuagesimus $=$ r, bl. $129^{\mathrm{b}}$ nach v. 3250 ; 2. Dialogus bl. g $4^{\mathrm{a}}$, gehörend zu dem De ornice et galliua dyalogus septuagesinus primus ibersch!riebenen capitel $=r$, bl. 130" nach i. 3254

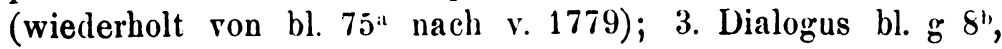
unter der überschrift: De turture casta dialogus septuagesiuus octauus $=$ r, bl. $130^{\prime \prime}$ nach r. $3258 ; 4$. Dialogus bl. f. $6^{\text {b }}$, unter der iiberschrift: De coruo et ficedula dyalogus sexagesimus tercius $=$ r, bl. $130^{\mathrm{b}}$ nach v. $3262 ; 5$. Dialogus bl. e $6^{\mathrm{a}}$, unter der überschrift: De herodio et miluo dyalogus quinquagesimus primus $=$ r, bl. $130^{\prime \prime}$ nach r. $3266 ; 6$. Dialogus bl. e $8^{b}$, unter der überschrift: De falcone et gallo dialogus quinquagesimus quintus $=$ r, bl. $131^{\text {: }}$ nach v. $3270 ;$ 7. Dialogus bl. g 4", unter der iiberschrift: De qlia et alauda dyalogus septuagesimus secundus $=\mathrm{r}$, bl. $131^{\mathrm{a}}$ nach v. 3274 . - Ausserdem kommen im R. V. noch folgende nachschnitte von holzschnitten des dialogus vor: 1. Dialogus bl. f $5^{a}$, gehörend zu dem De gallo et capone dyalogus sexagesimus primus ïberschriebenen capitel $=\mathrm{r}$ bl. $45^{\mathrm{b}}$ vor der capitelüberschrift $\mathrm{zu} \mathrm{I}, 13 ; 2$. Dialog. bl. f. $2^{a}$, unter der uberschrift: De osmerillo et accipitre dyalogus quinquagesimus septimus $=\mathrm{r}$, bl. $75^{\mathrm{b}}$, vor der capitelüberschrift zu I, 20; 3. Dialor. g 1" ciconia et yrundine dyalogus sexagesimus septimus $=r$, bl. $76^{\mathrm{a}}$, nach v. $1796 ; 4$. Dialogus bl. b $6^{\mathrm{b}}$, unter der liberschrift: De leone qui pugnauit cum aquila dyalogus octuagesimus quintus $=\mathrm{r}$, bl. $138^{\mathrm{a}}$ nach der capiteluberschrift zu II, 3; 5. Dialogus bl. b $3^{\prime \prime}$ unter der uberschrift: De smaragdo et anulo Dyalogus quartus decimus $=\mathrm{r}$, bl. $181^{\mathrm{b}}$ vor der capitelüberschrift zu III, 6; 6. Dialogus bl. b (6", gehörend zu dem De carbunculo et speculo Dyalogus decimus septimus uberschriebenen capitol $=$ r, bl. 184a, nach v. 4938 .

Da nun beide werke, sowol der Dialogus creaturarum als auch der Reinaert des Hinrek van Alckmer in derselben Leeuschen officin und dieser später als jener gedruckt worden sind, so ist es höchst warscheinlich, dass diese holzschnitte des Dialogus im druck des Reinaert wider gebraucht wurden und dass demgemäss sowol die vv. 3217 -3274 als auch die dazu gehörige inhaltsangabe in der vorrede des zweiten buches schon in der vorlage you $r$ standen. 
In diesem zusammenhange will ich nicht unterlassen za erwähnen, dass auch die holzschnitte des bruchsticks von $r$ verkleinert nachgeschnitten worden sind. Hoffmanns facsimile, bei dessen auswahl wol der umstand massgebend gewesen ist, dass dieser der einzig vollständig erhaltene bolzschuitt ist, gibt nun den holzschnitt wider, der gerade nicht in $\mathbf{r}$ aufgenommen wurde, wol aber sind es die beiden anderen: d, bl. $3^{\mathrm{b}}=\mathrm{d}$, bl. $4^{\mathrm{a}}$ ist nachgeschnitten in r, bl. 69", nach der uberschrift $\mathrm{zu}$ I, 18; und d, bl. $6^{\mathrm{a}}=$ r, bl. $72^{\mathrm{a}}$ zu I, 19 nach v. 1687 (und widerbolt bl. $164^{\mathrm{a}}$ zu III, 1 nach v. 4261). - Bindende beweiskraft für die annahme, dass $r$ direct $d$ benutzte, haben diese holzschnitte jedoch nicht - sie könnten in einer neuen auflage von $d$, nach welcher der nd. ubersetzer vielleicht arbeitete, widerholt sein (vgl. s. 34 u. 40).

\section{Zählung und einteilung der capitel.}

a. Zählung. Die nur in $r, d, b$ vorgenommene capitelzählung ist wenig geeignet, uns aufschluss über das verhältnis der nd. übersetzung zum original zu geben. $d$, von dem nur 5 (?) [nämlich die 17 (?), 19 (?), 22, 23, 24; s. o. s. 20] capiteleinteilungen erhalten sind, stimmt in seiner zählung weder uberein mit $r$ noch mit $h: d$ cap. $17(?)=r, I, 17 ;$ b, cap. 17; [d, cap. 18 (?) lässt sich nicht vergleichen]; d, cap. $19(?)=$ h, cap. 19 [r, v. 1577]; [d, cap. 20 (?)] = r, I, 18; b, cap. 20; [d, cap. 21 (?) lässt sich nicht vergleichen]; d, cap. $22=\mathrm{r}, \mathrm{I}$, 19 ; b, cap. 21 ; [d, cap. 23 vergleicht sich nicht]; d, cap. $24=$ r, I, 20; h, cap. 22 [im text ist xxiij druckfehler].

Schon hieraus erhellt, dass $h$ sich keineswegs in ubereinstimmung befindet mit $r$ und wenn trotzdem die 75 capitel in $\mathrm{r}(39+9+14+13)$ durch die 69 capp. in h (h zählt zwar 70 capitel, die ziffer 22 ist aber uberschlagen) beinahe erreicht werden, so ist daraus ebensowenig ein schluss zu ziehen, als aus der durch hinzunahme der in $h$ unbezifferten 7 abschnitte (ss. 7,$1 ; 8,21 ; 9,10 ; 30,6 ; 50,9 ; 53,16 ; 59,1$ ) gewonnenen annähernden ubereinstimmung. Dass uberhaupt auf die zählung nicht allzu grosse sorgfalt verwandt wurde, sieht man schon aus dem umstande, dass in $h$ jene 7 abschnitte von derselben ausgeschlossen und dass im register (Martin, Volksbuch s. 3) oft mehrere capitel unter einer tiberschrift zu- 
sammengefasst werden: so das II und III, LIIII-LIX; das XIV (wo im register XV druckfehler) hat im register uberschrift, im text s. 32 fchlt sie; überschlagen werden im register in der zählung die ziffern 18, 20, 24, 27, 28, 29, 33, 36, 38, $42,46,49,50,51,53$; zu capitel 52 werden im register zwei uberschriften gesetzt. - Auch R. V., wiewol in der kurzen tafel s. $22 i$ jedes mal genau die zabl der zu jedem buch gehörigen capitel angegeben werden, zählt nicht sorgfältig: auf s. 69 wird die uberschrift (vgl. damit den absatz in p, XXVIII ${ }^{a}$ ) ganz in der zählung ausser acht gelassen, womit sich freilich der glossator s. 70, 1; 70,17 in übereinstimmung befindet (die Ausgg. v. 1517 und 1539 tilgen die überschrift) und man muss sich wundern, zu anfang des zweiten buches keine capitelzäblung zu finden, da doch die überschrift vorhanden ist und die verse $3247-3274$ ein völlig abgeschlossenes ganze bilden.

b. Einteilung. Wenn die fortsetzung des ursprunglichen Willemschen, durch abgeschlossenheit und einfachheit sich auszeichnenden gedichtes schon an und für sich als eine im ganzen recht ungeschickte, in unnötiger breite sich ergehende widerholung tadel verdient, so kann man noch weniger die einteilung in vier bücher und die capiteleinteilung loben; denn die abteilung des ursprunglichen gedichtes als erstes buch sowie die einteilung der fortsetzung in drei blicher entbehrt jeglichen grundes und die capiteleinteilung fällt an vielen stellen so plump mitten in die er\%ählung hinein, dass ich kaum nötig habe, dafur noch beispiele anzufuhien.

Line genaue vergleichung, wie sie die angefugte tabelle (8. 34 f.) bieten soll, zeigt denn auch, dass man in allen hier in betracht kommenden gedicliten und auszugen sich wenig um die einmal getroffene einteilung kummerte, vielmehr oft einen abschnitt ansetzte, wo andere diesen nicht baben: ähnliche beobachtungen lassen sich auch bei anderen gedichten aus jener zeit anstellen.

Allein so gross die abweichungen in der einteilung in $p$, d, r, h scheinen, so wenig sind sie es in wirklichkeit, wenn wir, was füglich erlaubt ist, diejenigen stellen mitrechnen, in denen in p zwar keine durch eine iberschrift gekennzeichnete einteilung, wol aber ein absatz gemacht worden ist, der einen sinnabschnitt andeutet und einer wirklichen einteilung in $r$ 
oder $\mathrm{h}$ entspricht. Es würden demnach zu den 1 (columne I) +1 (col. II) +11 (col. IV) $=13$ fällen, in denen $\mathrm{p}, \mathrm{r}, \mathrm{h}$ einen mit einer überschrift (und, soweit sie uberhaupt zählen, auch mit bezifferung) versebenen absatz machen, noch hinzukommen 2 (col. V) +1 (col. III) +16 (col. IX) $=19$ stelleu, zusammen 32 einteilungen; von den in $r$ ubrig bleibenden 43 einteilungen hat es mit $\mathrm{p}$ gemeinsan 9 (col. VI), mit h 4 (col. IX, nr. 2, 5, 12, 19), während $r$ an 2 stellen einteilt, wo $p$ und h einen absatz machen (col. XIII, nr. 1 und 12) und an 9 stellen (col. XIII), wo p allein absetzt $=$ zusammen 24 einteilungen, so dass wir nur an 19 stellen $r$ eine selbständige einteilung machen sehen. Hierbei ist jedoch noch zu berücksichtigen, dass an 2 stellen r, II, 8 und r, II, 9 (col. XIII) überschriften $z u$ abschnitten stehen, die von $r$ umgearbeitet zu sein scheinen. Man sieht hieraus, dass $p$ mit seinen uberschriften und seinen absätzen grundlage war für alle späteren einteilungen; die geringen abweichungen von derselben in $r$ mögen teilweise schon in des letzteren quelle gestanden haben, teilweise durch die raumverhältnisse der Llibeker ausgabe gefordert worden sein. -

Eine von dieser abweichende einteilung nebst überschriften (aber ohne capitelzählung) ist schon in dem vor 1280 verfassten 1 vorhanden; dieses, nur die lateinische tibersetzung des ursprünglichen Willemschen gedichtes enthaltend, teilt an $\delta$ stellen ein (col. I, III u. X) und nur an 1 stelle (col. I) stimmt es mit $p, r$, h zusammen, während $p$ an einer zweiten stelle, wo 1 mit $r$, h stimmt (col. III) nur einen absatz macht. Daraus geht augenscheinlich hervor, dass 1 demjenigen, der die einteilung furr $p$ vornalım, nicht bekannt gewesen oder wenigstens von ibm nicht beachtet ist; die thereinstimmung in der einteilung an jenen beiden stellen wird ganz zufällig sein.

\section{Capiteluberschriften.}

Wichtiger für die ubersetzertätigkeit als alle bisher angefuhrten pnnkte sind die capiteluberschriften.

Es ist seit dem glucklichen funde, der uns das bruchstuck d zugänglich gemacht hat, behauptet worden, dass uns in demselben ein teil der vorlage des R. V. gerettet sei, auch hinsichtlich der uberschriften. Eine vergleichung lehrt, dass nur 
in einem einzigen falle $\left(\mathrm{p}, \mathrm{XXIII}^{\mathrm{b}}=\mathrm{d}\right.$, cap. $22=\mathrm{r}, \mathrm{I}, 19=$ h, cap. 21) r zu d stimmt. Die frage wird sich zur entscheidung bringen lassen mit hulfe von h (wenig in betracht kommen kann $p$, da in diesem die capitel nur höchst spärlich taberschriften erhalten haben): können wir éine überschrift nachweisen, wo h mit $r$ gegen d stimmt, so sind wir berechtiogt, $d$ als directe quelle für die aberschriften in $r$ abzuweisen. Dies ist nun allerdings, soweit wir nach dem erhaltenen urteilen können, nicht der fall, und, nachdem wir im stande gewesen sind, die defecten stellen in $d$ zu ergänzen, nötigen uns auch diese ergänzungen nicht, fur $\mathrm{r}$ eine andere quelle zu suchen, obgleich wir oft erst mit htilfe von $h$ einer ibberschrift an den defecten stellen ibren platz angewiesen haben - aber doch nur erst dann, wenn die raumverhältnisse in d dazu fuhrten, wobei dann h erwunschte bestätigung einer ausgesprochenen vermutung bieten konnte.

Die erste vergleichbare uberschrift ist d, eap. 19 (?) = h, cap. 19; diesclbe weicht in h deshalb von $d$ ab, weil ersteres den schluss der beichte (R. II, 1655-1681) ausgelassen hat und gleich zur absolution durch Grimbart ubergegangen ist. r hat die uberschrift ganz unberlicksichtigt gelassen, auch in der uberschrift zu I, 17, zu welchem capitel die erzählung von der beichte gehört.

Dic $\mathrm{zweite}$ überschrift stimmt in allen 4 recensionen im wesentlichen uberein $\left(\mathrm{p}, \mathrm{XXIII}^{\mathrm{b}}=\mathrm{d}\right.$, cap. $22=\mathrm{r}, \mathrm{I}, 19=$ h, cap. 21).

Die dritte aberschrift d, cap. 23 haben sowol $r$ als h ausgelassen. $r$ kurzt uberhaupt zu anfang des 19. capitels, indem den 54 versen in R. II $(1771-1824) 37$ verse in $\mathrm{r}(1685-1722)$ entsprechen. Fur das verfahren in $r$ und $b$ ist der grund leicht einzusehen, denn die uberschrift d, cap. 23 fuhrt dasjenige nur breiter aus, was schon in der uberschrift d, cap. 22 gesagt worden ist.

Die vierte uberschrift ist $\mathrm{p}, \mathrm{XXII^{n }}$ rect. $\mathrm{XXV}^{a}=\mathrm{d}$, cap. $24=\mathrm{r}, \mathrm{I}, 20=\mathrm{h}$, cap. 23. Hier stimmen (p) $\mathrm{d}$ und h uberein, während $r$ den gleichen inhalt aber anderen wortlaut hat.

Ich gehe zu den für d erschlossenen uberschriften über. Die erste d, cap. 18 (?) wurde sich mit h, cap. 18 vergleichen lassen; r konnte sie füglich nicht berticksichtigen, da sie nur eine unterabteilung zu r, I, 17 gebildet haben würde. 
In der zweiten erschlossenen überschrift d, cap. 20 (?) stimmt r, I, 18 mit h, cap. 20 und p, XXII" macht einen absatz.

$\mathrm{Zu}$ der dritten iberschrift d, eap. 21 (?) können wir keine aus den andern recensionen vergleichen, auch $\mathrm{p}, \mathrm{XXIII"} \mathrm{macht}$ keinen absatz.

Hiernach hat also $r$ drei uberschriften mit d gemeinsan, indem es in der einen $r, I, 19=d$, eap. 22 (und vielleicht auch in der andern r, I, $18=\mathrm{d}$, cap. 20 (?)) wörtlich mit d ubereinstimmt, während es in der dritten r, I, $20=d$, cap. 24 im wortlaut von $d$ abweicht. Die vier übrigen iiberschriften hat $r$, da sie nur unterabteilungen vorhergegangener überschriften waren, ausgelassen und so unter I, 17 die uberschriften d, cap. 18 (?) und 19 (?), unter I, 18 die uberschrift d, cap. 21 (?) und unter I, 19 die überschrift d, cap. 23 zusammengefasst.

Die iibereinstimmung von 1 in einer uberschrift $(1,798=$ p, XXIII" $^{\prime}=$ d, cap. $22=$ r, I, $19=$ h, cap. 21) wird auch hier nur zufällig sein.

Es wird demgemäss zur höchsten wahrscheinlichkeit, dass $r$ in seinen capitelïberschriften $d$ als quelle benutzte; bis zur evidenz kann das freilich nicht bewiesen werden, da uns der geringe umfang von $d$ nur eine beschränkte vergleichung gestattet und es bleibt immer noch die möglichkeit bestehen, dass d noch einmal einer redaction unterzogen wurde, in der es eine sich $r$ mehr nähernde fassung erhielt, und dass dann diese, alsu cine neue auflage von $d$, dem ubersetzer von $r$ vorlag.

Uebersichtliche vergleichung der capitel-einteilungen ')

(zu s. 31 ff.).

I, l, p, d, r, h.

l, $798=$ p, $\times X 111^{b}=d$, cap. $22=$, $1,19=$ h, cap. 21 .

II. $p, d, r, h$.

p, XXIIa rect. XXVa $=$ d, cap. $24=$ r, I, $20=$ h, cap. $23 . \quad l, 884$.

1) Diese tabelle soll eine übersichtliche vergleichung der capiteleinteilungen der verschiedenen recensionen bieten. $\mathrm{Zu}$ den zwecke sind auf der jeweiligen linken columne diejenigen stellen verzeichnet, wo eine solche, verbunden mit iiberschrift und, wo gezählt wird, auch mit bezifferung gemacht worden ist; die rechte columue gibt die stellen an, 
III. $\mathrm{l}, \mathrm{r}, \mathrm{h}$.
l, $1490=\mathrm{r}, \mathrm{I}, 34=\mathrm{h}$, cap. 34 .
$p^{*}, X X X 1 X^{a}$.

IV. $p, r, h$.

1) $\mathrm{p}, \mathrm{I}^{\mathrm{b}}=\mathrm{r}, \mathrm{I}, 2=\mathrm{h}$, cap. 1 .

l, 37.

2) $\mathrm{p}, \mathrm{III}=\mathrm{r}, \mathrm{I}, 3=\mathrm{h}$, cap. 2 .

$l, 89$.

3) $\mathrm{p}, \mathrm{VI}^{\mathrm{b}}=\mathrm{r}, \mathrm{I}, 5=\mathrm{h}$, cap. 6 .

4) $\mathrm{p}, \mathrm{XIVa}=\mathrm{r}, \mathrm{I}, 12=\mathrm{h}$, cap. 12 .

$l, \cdot 181$.

5) p, XXXVIII" $=\mathrm{r}, \mathrm{I}, 32=\mathrm{h}$, cap. 32.

6) $\mathrm{p}, X L^{b}=\mathrm{r}, \mathrm{I}, 35=\mathrm{h}$, cap. 35 .

$l, 432$.

$[l, 1427$.

l, 1544.

7) $\mathrm{p}, \mathrm{XLVa}=\mathrm{r}, \mathrm{I}, 39=\mathrm{h}$, cap. 39 .

8) p, $\operatorname{XLIX}^{\mathrm{b}}=\mathrm{r}$, II, $4=\mathrm{h}$, cap. 43.

9) p, LVIIIa $=$ r, III, $2=$ h, cap. 46.

1ii) $\mathrm{p}, \mathrm{m}$ VI' $=\mathrm{r}, \mathrm{IV}, 5=\mathrm{h}$, cap. 66 .

11) p, n I" $=$ r, IV, $\tau=h$, cap. $6 \tau$.

V. d, r, h.

1) d, cap. 17 (?) $=$ r, I, $17=$ h, cap. 17 .

2) d, cap. 20 (?) $=$ r, 1, 18 = h, cap. 20.

$[l, 646] ; p^{*}, X X^{a}$.

$l, 766 ; p^{*}, X X I l^{b}$.

VI. $p, r$.
1) $\mathrm{p}, \mathrm{Va}=\mathrm{r}, \mathrm{I}, 4$.
2) $p, X L I I "=r, I, 37$.
3) $\mathrm{p}, \mathrm{XLVb}=\mathrm{r}, \mathrm{II}, 1$.
4) $\mathrm{p}, \operatorname{XLVIII} a=r, I I, 2$.
5) $\mathrm{p}, 1$ VII' $=\mathrm{r}, \mathrm{IV}, 1$.
(i) p, m VII" unten $=$ r, IV, 6.
7) $\mathrm{p}, \mathrm{n} \mathrm{II}^{\mathrm{a}}=\mathrm{r}, \mathrm{IV}, 8$.
8) $\mathrm{p}, 0 \mathrm{I}^{\mathrm{b}}=\mathrm{r}, \mathrm{IV}, 10$.
9) $\mathrm{p}, \mathrm{o} \mathrm{III}^{\mathrm{b}}=\mathrm{r}, \mathrm{IV}, 11$.

$l, 135 ; h, 14,16$.

$l, 1656 ; h, 62,26$.

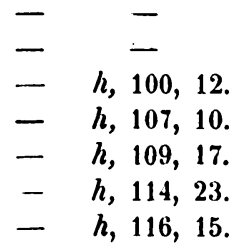

VII. $p, h$.

1) $\mathrm{p}, \mathrm{II} \mathrm{a}=\mathrm{h}^{\dagger}$, s. 8 .

2) $\mathrm{p}, \mathrm{II} \mathrm{a}=\mathrm{ht}$, s. 9 .

3) $\mathrm{p}, \mathrm{VII}^{\mathrm{b}}=\mathrm{h}$, cap. 7 .

4) $\mathrm{p}, \mathrm{IX}^{\mathrm{a}}=\mathrm{h}$, cap. 9 .

$l, 53 ; \quad r, 69$.

$\ell, 5 i ; \quad r, i 8$.

$l, 211 ; r, 471$.

$l, 249 ; r, 571$.

an welchen eine einteilung der links verzeichneten entsprechen würde. Ein * bei $\mathrm{p}$ und $\mathrm{l}$ deutet an, dass ein absatz bezw. ein grosser buchstabe zu verzeichnen ist; mit einem + sind solche einteilungen versehen, welchen iberschrift oder bezifferung oder beides mangelt. $d, r, h$ sind nach capiteln, 1 (wo nötig auch r) nach versen, $p$ nach blättern, $h$ wo nơtig auch nach seiten und zeilen angeführt. In [] sind solche stellen geschlossen, die eine directe vergleichung ausschliessen. 
VII. $p, h$.

\begin{tabular}{|c|c|}
\hline 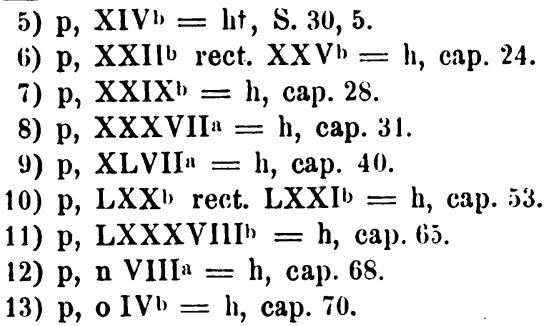 & $\begin{array}{c}l, 452 ; r, 949 . \\
l, 914 ; r, 1851 \\
l, 1064 ; r, 212 \pi \\
l, 1402 ; r, 2622 . \\
-\quad ; r, 3350 . \\
-\quad ; r, 4515 . \\
-\quad ;[r, 5539 .] \\
-\quad ; r, 6517 . \\
-\quad ; r, 6740 .\end{array}$ \\
\hline
\end{tabular}

VIII. d, h.

1) $\mathrm{d}$, cap. 18 (?) $=h$, cap. 19 .

2) d, cap. 19 (?) = h, cap. 19.

$l, 694 ; p, X X I^{\iota} ; r, 1534$.

$l, 758 ; p, X X 11 a ; r, 1592$.

IX. $r, h$.

1) $r, I, 7=h$, cap. 8 .

2) $\mathrm{r}, \mathrm{I}, 11=\mathrm{h}$, cap. 11 .

3) $r, I, 13=h$, cap. 14 .

4) $r, I, 15=h$, cap. 16 .

5) r, I, $22=$ h, cap. 25 .

6) r, I, $24=$ h, cap. 27 .

7) $\mathrm{r}, \mathrm{I}, 2 \mathrm{i}=\mathrm{h}$, cap. 29.

8) r, I, $30=h$, cap. 30 .

9) $r, 1,33=h$, cap. 33 .

10) $\mathrm{r}, \mathrm{I}, 38=\mathrm{h}$, cap. 35 .

11) $\mathrm{r}, \mathrm{Il}, 3=\mathrm{h}$, cap. 42 .

12) r, III, $1=h$, cap. 45 .

13) r, III, $6=$ h, cap. 54 .

14) r, III, $7=$ b, cap. 55 .

15) r, III, $8=$ h, cap. 56 .

16) $r$, III, $9=$ h, cap. 57 .

17) $\mathrm{r}, 1 \mathrm{II}, 10=\mathrm{h}$, cap. 58 .

15) r, III, $11=$ h, cap. 59.

19) r, III, $13=$ h, cap. 61 .

20) $r, I V, 2=h$, cap. 64 . $\left.l, 2: 35 ; p^{*}, V I I I a\right]$.

$l, 412 ; p, X I I l^{l}$ mitte.

$l, 518 ; p^{*}, X V 1^{b}$.

$l, 602 ; p^{*}, X 1 X^{a}$.

$[l, 946] ; p, X X \vee 1 I^{a}$.

$[l, 1038] ; p^{*}, X X 1 X a$.

$l, 1196 ; p^{*}, X X X I 1 I^{a}$.

$l, 1360 ; p^{*}, X X X V I^{a}$.

$l, 1455: p^{*}, X X X V 111^{b}$.

$l, 1730 ; p^{*}, X L 1 V a$.

- ; $p^{*}, X L V I H^{b}$.

- ; p, LVILa.

- ; $p^{*}, L X X I^{b}$ rect. LXXIIb.

- ; $p^{*}, L X X I I I a$ rect. LXXIVa.

- i $p^{*}, k, V^{b}$.

- ; $p^{*}, L X X V I^{b}$, recl. LXXVIIb.

- ; $\nu^{*}, L X X V I I^{b}$, rect.L.XXVIII".

- ; $p^{*}, L X X I X^{b}$.

-- ; p, LXXX11b.

- ; $p^{*}, l, V I I I b$.

X. 1 .

\begin{tabular}{l|ll}
\hline 1$) 1,197$. & $p, V I I a ;$ & $r, 446 ; h, 18,21$. \\
2) $1,426$. & $p, X I V a$ ob.; & $r, 896 ; h, 28,22$. \\
$3) l, 584$. & $p, X V I I I^{b}$ ob.; & $r, 1281 ; h, 35,6$. \\
4) $1,980$. & $p, X X V I I b$ mitt.; r, $1960 ; h, 45,23$. \\
5) $1,1020$. & $p, X X V I I I a$ unt. $r, 2031 ; h, 46,25$. \\
6) $1,1370$. & $p, X X X V I b$ ob.; r, $2581 ; h, 54,25$. \\
\hline
\end{tabular}


XI. p.
1) p, XIII".
2) p, XVIII".
3) $\mathrm{p}, \mathrm{XIXh}$.
4) p, XXVII".
5) $\mathrm{p}, \mathrm{L}^{\mathrm{b}}$.
b) p, LVIIa.
7) p, LXIII".
s) p, LXV".
9) p, LXIX".
10) p, in VIIa ob.
11) p, n IVb.

$$
\begin{gathered}
l, 418 ; r, 884 ; h, 28,15 . \\
l, 578 ; r, 1275 ; h, 35,2 . \\
l, 622 ; r, 1371 ; h, 36,21 . \\
{[l, 978] ; r, 1972 ; h, 45,17 .} \\
-\quad r, 3587 ; h, 70,26 . \\
-\quad ; r, 4264 ; h, 74,24 . \\
-; r, 4532 ; h, 82,9 . \\
-; r, 4579 ; h, 83,3 . \\
-\quad-\quad-6138 ; h, 107,5 .
\end{gathered}
$$

XII. d.

1) d, cap. 21 (?).

2) d, cap. 23 . $l, 786 ; p, X X I I l a ; r, 1665 ; h, 40,22$.

$l, 828 ; p^{*}, X X I I I$ a rect. $X X 1 V a ; r, 1723 ; h, 41,27$.

XIII. $r$.
1) $\mathrm{r}, \mathrm{I}, 1$.
2) $r, I, 6$.
3) $\mathrm{r}, \mathrm{I}, \mathrm{s}$.
4) $r, I, 9$.
5) $\mathrm{r}, \mathrm{I}, 10$.
6) $\mathrm{r}, \mathrm{I}, 14$.
7) $\mathrm{r}, \mathrm{I}, 16$.
5) r, I, 21.
9) r, I, 23.
10) r, I, 25.
11) r, I, 26.
12) r, I, 28.
13) r, I, 29.
14) r, I, 31 .
15) $\mathrm{r}, \mathrm{I} ; 36$.
16) $r+$ II.
17) r, II, 5 .
18) r, II, 6.
19) r, II, 7 .
20) r, II, 8 .
21) r, U, 9.
22) r, III, 3.
23) r, III, 4.
24) r, III, 5.
25) r, III, 12.
26) r, III, 14.
2i) r, IV, 3.
$l^{*}, 23 ; p^{*}, I^{b} ; h+, 7,1$.
$l, 203 ; p, V I I a$ mitte; $h, 19,1$.
$l, 275 ; p, I X^{b} ; h, 24,2$.
$l, 297 ; p^{*}, X^{b} ; h, 25,14$.
$l, 368 ; p^{*}, X I^{b} ; h, 26,17$.
$l, 518 ; p, X V I^{b}$ unt.; $h, 33,1$.
$l, 610 ; p^{*}, X 1 X^{b} ; h, 36,10$.
$l, 886 ; p, X X 11$ a rect. $X X V a ; h, 43,7$.
$l, 1027 ; p, X X V I 1 l^{b} ; h, 47,6$.
$l, 1134 ; p^{*}, X X X I b ; h, 51,3$.
$[l, 1160] ; p^{*}, X X X I I^{b} ; h, 51,18$.
$l, 1234 ; p^{*}, X X X I V a ; h \dagger, 53,16$.
$l, 1276 ; p^{*}, X X X \perp V^{b} ; h, 53,26$.
$[l, 1384] ; p, X X X V I I a ; h, 55,6$.
$l, 1631 ; p^{*}, X L I I^{b} ; h, 62,8$.

$$
\begin{aligned}
& \text { - } \quad p, \overline{L l} ; \boldsymbol{L}, \mathrm{il}, \overline{7} \text {. } \\
& \text { - ; } p, L I I^{b} \text { mitte; } h, i 1,23 . \\
& \text { - ;p, } L I V^{b} ;[h, 74,5] \text {. } \\
& \text { - ; }\left[p, \bar{L}\left[X^{a}\right] ;[\bar{h}, \bar{\tau}]\right. \text {. } \\
& \text { - ; } p^{*}, L X I I^{b} ; h, 80,22 . \\
& \text { - ; [p, LXVb]; [h, 83,3]. } \\
& \text { - ; p, LXXa rect. LXXIa; - } \\
& \text { - ;p,lVIb;h, 99, } 7 \text {. } \\
& \text { - ; [p, LXXXVIIIb milte]; }[h, 102,20] .
\end{aligned}
$$$$
\text { - ; p, LXXXla; } h, 95,25 .
$$ 
XIII. $\mathbf{r}$.

28) r, IV, 4 .

29) $r$, IV, 9.

30) r, IV, 12.

31) r, IV, 13.

- ; p, m 11b; h, 103, 1.

- ; $p, n$ VIIIa mitte;

- ; p, o IVa; $h, 117,5$.

- ; $p^{*}$, o $V l^{\alpha} ; h, 118,1$.

XIV. h.

1) $h+$, s. 12.

2) h, cap. 3 .

3) h, cap. 4 .

4) h, cap. 5 .

5) h, cap. 10 .

6) h, cap. 13 .

7) h, cap. 15 .

8) h, cap. 26.

9) $h \dagger$, s. 50 .

10) $h+$, s. 59,1 .

11) h, cap. 36 .

12) h, cap. 37 .

13) h, cap. 41.

14) h, cap. 44.

15) h, cap. 47 .

16) h, cap. 48 .

17) h, cap. 49.

18) h, cap. 50 .

19) h, cap. 51 .

20) h, cap. 52.

21) h, cap. 60.

22) h, cap. 62.

23) h, cap. 63.

24) h, cap. 69 . $l, 111 ; p^{*}, 111^{b} ; r, 227$.

$l, 117 ; p, I V a ; r, 247$.

$l, 131 ; p, 1 l^{r} b$ unt.; $r, 293$.

$[l, 171] ; p^{*}, V^{b} ; r, 348$.

$l, 294 ; p, X^{b} ; r, 657$.

$l, 503 ; p^{*}$, IVIa $r, 1041$.

$l, 566 ; p, X I I I I a ; r, 1236$.

$l, 1007 ; p^{*}, X X V 1 I 1 a ; r \dagger, 2021$.

$l, 1118 ; p^{*}, . X X X I a ; r, 2218$.

$l, 1527 ; p, X L a$ mitte; $r, 2803$.

$l, 15 \mathrm{~S} 2 ; p^{*}, \mathrm{XLIa} ; r, 2596$.

$l, 1694 ; p^{*}, X L I 1 l^{b} ; r, 3089$.

— ; $p, X L V I a ; r, 3307$.

- ; $p, L I 1 a$ mitte; $r, 3672$.

- ; $p^{*}, L I X^{b} ; v, 4384$.

- i $p, L X a$ mitte; $r, 4407$.

- ; $p^{*}, L X I a ; \quad$ -

- ; $p, L X I I a$ mitte; $r, 4436$.

- ; $p, L X I 1 L a ; r, 4480$.

- ; $p, L X I 11 b ; r, 4541$.

- ; $p^{*}, L X X X^{a} ; r, 5253$.

- ; p,l VIa; r, 5491.

- ; $p, l$ VIIb ob.; $r, 5596$.

- ; $p, o$ Ia mitte; $r, 6587$.

\section{Die glosse.}

Bis vor nicht langer zeit hat man noch die sogenannte katholische glosse des R. V. für ein charakteristicum der nd. ubersetzung gehalten; so noch J. Grimm R. F. s. CLXXV anm.; dieser auffassung ist Latendorf, Programm des Schweriner Gymnasiums 1865 s. 31 bestimmt entgegen getreten, nachdem Hoffmann in der ersten auflage seiner ausgabe des R. V. s. XXII die gegenteilige ansicht bloss hypothetisch ausgesprochen batte. Freilich nachdem (zuerst durch Latendorf, a. a. 0. s. 30) die volksbücher in die untersuchung hineingezogen worden sind, 
ist auch daran nicht der leiseste zweifel gestattet. Aber mich däucht, eine genaue einsicht in die worte der nd. glosse hätte schon vorher zu demselben resultat führen können, nachdem es ausgemacht war: dass Hinrek van Alckmer nicht der verfasser der nd. ibbersetzung sein konnte; mit den worten unde hebbe bi islik capittel gesat eine korte atlegginge unde meninge des sulfsten poeten (Lübben s.IV, 6,7 ) ist deutlich genug gesagt, dass der Niederlïnder, wenn auch nur eine korte glosse dem gereinten text beigefiigt hat. Verwirrung ist dadurch in die sache hineingekommen, dass der nd. ïbersetzer seine glosse in diejenige des Hinrek van Alckmer hineingeflochten hat, obne dieselbe jedesmal mit klaren worten als sein eigentum zu bezeichnen, sowie dadurch, dass er gleicherweise den eigentlichen dichter und den nl. glossator mit meister, lerer bezeichnet $z$. $b$. s. 109, 12 darin leret de lerer (der verfasser des Reinaert) und beslut darmit dat êrste bôk (der nl. glossator). Dieser nl. ûtlegginge stellt der nd. iibersetzer seine eigene nur zweimal bestimmt gegenuber, indem er den nl. glossator vom dichter treunt: cap. II; 9 s. 142, I Van dessen secht he nicht vele in desser âtlegginge und noch deutlicher: cap. III, 12, s. 179 Up dit vorgesechte capittel is sunderlik nene atlegginge gesat ....

Wenu so fest steht, dass der niederdeutsche in seiner vorlage eine glosse schon vorfand, so bleibt noch $\mathrm{zu}$ untersuchen, hat er eigene zusïtze gemacht, welche sind diese und ist eine entscheidung daruber möglich, welchem nl. original er gefolgt ist.

Gehen wir auf die letzte frage zuerst ein. Es sind hier nur $d$ und $h$, welche mit $r$ verglichen werden können. Das bruchstlick uberliefert uns nur an 2 stellen glossen, die näher zu betrachten sind.

Die erste glossiert d, cap. 17 (?) und stimmt, soweit sie erhalten ist, mit $\mathrm{h}$ zu cap. 17 und r, I, 17 s. 54, 8 ff. uiberein, nur dass in letzterem allgemeine ausdrucke, wie $\mathrm{d}=\mathrm{h}$ die ghierighen und d heeren ende vrouwen specialisiert werden durch alle de, dede komen bi ein lên efte provene, vogedie, efte wal it si, dar rente efte vordèl to boren is, edder $\hat{o} k$ ein ander giriger, .bezw. dat he heft gedân untruwe unde schande sinem heren, deme konninge unde der konninginnen. Auch ist die reihenfolge der glossierung in $r$ eine andere als in $d$ und von der breiten aus- 
flihrung des dritten punktes in $r$ s. 54, $21-56,9$ ist in d nichts zu finden.

Schwieriger ist die $\mathrm{zw}$ eite glosse zu d, cap. 23; von ibr sind zwei im anfange verstummelte zeilen tuberliefert. $h$ hat bier keine moralisation. $r$ fasst in der glosse s. 63 die capitel I, 19 und I, 20 zusammen, und zieht aus ihnen funf lehren. Die funfte nutzanwendung s. 63,.10 ff. ist aus dem capitel I, 20 entnommen ( $\mathrm{zu}$ welchem das entsprechende capitel in d nicht mehr erhalten ist), die vier ersten lehren glossieren das capitel I, 19, aber nur die vierte diejenigen verse (1723-1790), welche d, cap. 23 (fragm. v. 162-222) entsprechen: diese kann also hier nur in betracht kommen. Von den in d erhaltenen worten findet sich nun nicht ein einziges in $r$; deshalb aber schon d für die glossierung in $r$ abzuweisen, wäre unstatthaft, da $r$ geändert bezw. gekürzt haben kann.

Die oben erschlossenen glossen lehren uns vor allen dingen, dass auch hier nicht der fall eintritt, dass $r$ und $h$ glossierung haben, wo sie d fehlt; es wird daher auch hinsichtlich der glossen $d$ die grundlage für ${ }^{*}$ abgegeben haben, jedoch so, dass letzteres dieselbe je nach umständen zusammenzog (wie die glosse zu d, cap. 23) oder erweiterte (wie diejenige zu d cap. 17 (?)) oder mehrere capitel unter einer glosse zusammen betrachteté (wie glosse zu r, I, 20). Eine andere beobachtung ist die, dass in dem bruchstück jeder capiteluberschrift eine kurze moralische nutzanwendung angehängt wurde - wir werden berechtigt sein, dies auch auf die ganze bearbeitung auszudehnen, wodurch wir eins der merkmale der Hinrek van Alckmerschen bearbeitung erhalten haben (unde hebbe bi islik capittel eine korte itlegginge gesat). Auch für die glosse also wird d höchst wahrscheinlich als quelle für $r$ gelten mussen, doch ist auch hier wider wie bei den capiteltiberschriften (s. o. s. 34) die möglichkeit nicht ausgeschlossen, dass die glosse in $\mathrm{d}$ einer gelinden änderung unterzogen wurde, welche dann r vorgelegen haben könnte.

Eine art glossierung findet sich auch schon in 1 , indem kurze moralische lehren an erzählte vorgänge angeknlipft werden, die sich alle, ohne dass der zusammenhang eine störung erlitte, ausscheiden lassen (vgl. Schulze, uber Reinardus Vulpes ed. Knorr, Progr. d. Pädagogiums in Zullichau, Leipzig, 
1862 s. III). Latendorf a. a. o. s. 32, hat geglaubt, dass man an eine entlehnung aus 1 durch $d$ denken dürfe und hat für diese ansicht $1,796 / 7$ angefuhrt

Par huic est qui confessus sua probra nec horret

Ad mala quae planxit prima redire cito.

welche stelle sich fast wörtlich widerfinde r, gl. z. I, 18 s. 58 14-19: Liat vêrde is, dat mannich sunder sine sunde bichtet unde dar bote vor entfanget, men de ruwe is in em nicht wârhaftich; wente etlike sin, de bichten ere sunde und entfangen bote dar vor, men se beleven noch ellike vorgangen sunde, unde hebben. nene nàraftige ruwe vor alle unde sên to rugge, so Reinke hir dede na den hônren. An und fiur sich könnte sie freilich eine directe ubersetzung und erweiterung der angefühten lateinischen quelle sein, aber wenn wir im ubrigen die von Schulze a. a. o. verzeichneten moralischen lehren zwar dem inhalt, nicht aber der form nach in $r$ finden, es auch aus den oben (s. 32 und 34) berluhrten grlinden nicht wahrscheinlich ist, dass l überhaupt von Hinrek van Alckmer benutzt worden ist, so werden wir 1 als quelle fur die glossierung abzuweisen berechtigt sein; dass $l$ und $r$ in jenen worten zusammenstimmen, fordert die natur der sache: was lag näher, als den gedanken in der moralisation auszusprechen, dass der verstockte sünder trotz seiner reuigen busse dennoch immer wider in die alte sunde verfalle?

Wir haben schon oben gesehen, dass die moralisationen in $h$, wo sie mit $d$ verglichen werden können, sich mit diesem in ebenso grosser ubereinstimmung befinden, als bezliglich der uberschriften. Latendorf a. a. o. 8. 30 (der freilich h noch nicht, aber doch ein sicher aus demselben geflossenes [vgl. Martin, Volksbuch s. V] nl. volksbuch kannte) schliesst daraus, dass diese sowie auch der text in $b$ (mit $r$ ) direct auf d zurickgehe. Ihm widerspricht mit recht Martin, Reinaert s. XXVI und Volksbuch s. X und begrindet durch vorfuhrung der gemeinsamen abweichungen vom poetischen text die ansicht, dass $h$ auf $p$ zurlickgehe (vgl. auch Grimm R.F. s. CLXIV). Zur erklärung der aufnahme der moralisationen in $\mathrm{h}$ will mir die vermutung Martins a. a. o., dass aus einem exemplar von d dieselben in $h$, vielleicht mit einigen änderungen, eingetragen seien, recht 
annehmbar erscheinen: zugleich mag dann eine rovision der capiteliiberschriften vorgenommen sein.

Sonach werden wir füglich an denjenigen stellen, wo uns d im stiche lässt, $h$ zur vergleichung der glossen in $r$ heranziehen llirfen, wobei jedoch $z u$ beachten ist, dass $h$ zeitlich weit von r abliegt, und sich als volksbuch grössere kiirzungen erlauben konnte. Von den 40 moralisationen in $\mathrm{b}$ stehen folgende ohne vergleichung mit $\mathrm{r}:$ h s. 12,$5 ;$ s. 12,$20 ;$ s. 20,25 ; s. 37,$18 ;$ s. 54,$11 ;$ s. 58,$26 ;$ s. 64,$12 ;$ s. 66,$18 ;$ s. 80,4 ; s. 82 , 15 ; s. 94,$9 ;$ s. 95,7 ; s. 102,7 ; s. 117,13 ; von den tibrigen 26 lassen sich $18 \mathrm{dem}$ sinue nach mit $\mathrm{r}$ vergleichen: h, s. 24, 25 $=\mathrm{r}, \mathrm{I}, 11$, s. $29 ;$ b, s. $28,6=\mathrm{r}, \mathrm{I}, 11$, s. 29,$31 ; \mathrm{h}, \mathrm{s} .41,1=$ r, I, 18 , s. $58 ;$ h, s. $44,17=$ r, I, 22 , s. $70 ;$ h, s. $47,13=$ r, I, 22 , s. 70,$18 ;$ h, s. $48,19=$ r, I, 22 , s. 70,2 ; h, s. $50,15=$ r, I, 24 , s. 76,$2 ;$ h, s. $53,14=$ r, I, 31 , s. 89,$10 ;$ h, s. 55,11 $=\mathrm{r}$, überschrift I, 31 , s. 87 ; h, s. $59,14=\mathrm{r}, \mathrm{I}, 34$, s. 96, 1 ; h, s. $61,2=$ r, I, 34, s. 95 , 13; h, s. 77, $14=$ r, III, 2, s. 149, 9; h, s. $79,9=$ r, II, 9 , s. 141,9 ; h, s. $86,2=$ r, III, 4 , s. 160,21 ; h, s. 9 I, $22=$ r, III, 8 , s. 170,9 ; h, s. $93,2=$ r, III, 9, s. 172 ; h, s. $106,12=$ r, IV , 4, s. 203 ; h, s. $114,2=$ r, s. $188,13^{1}$ ) und IV, 9, s. 218. Die letzten acht stellen haben auch teilweise wörtliche ubereinstimmung:

\section{h}

(s. 9) Men sict ghemeenlijck in des Princen hof, dat de groote Heeren altijt ouer de slechte Edele te claghen hebben, ende so haest alsser - yemant van desen clachtich valt, so claghen oock lichtelijck met hem alle ander Heeren, van hoo leoghen state oft conditic dat sy ooc sijn. Naer tis seer goet ende orboorlijck eenen vriendt int hot te hebbe, die sy- (zu I, 3 3 8. 9, 2) ... wo de girigen in der heren hove vaken sake vinden van hate unde klagen over andere, de under en sin ...... ..... so wan ein grôt geachtet man over jemande klaget, dat denne ok vaken de kleinen beginnen to klagen over den sulven ....

1) Die ersten worte dieser moralisation finden sich auch in $r$ (aber an ganz anderer stelle); es wird das kaum gegen meine auffassung betreffs der vorrede zum vierten buch des R. V. (s. o. s. 27) sprechen, denn h glossiert hier den kampf Isegrims und Reinkes, wo der gedanke, dass schlauheit und list oft körperstïrke besiegt, sehr nahe lag: $r$ kürzt bedeutend die erzählung des kampfes. 
h

nen vrient in zijn absentie verantwoorde.

(s. 1i) Men behoort gheenen viant te gheloouen, in hoe schoonen schijnsel dat hy coemt. Men en sal oock ghcen lieden betrouvven die in heylighe cleederen, of $t$ onder tdecsel van heylicheyt comen, sprekende, ende hen beroemende van hen heylicheyt, want daer niet dan bedroch in gheleghen en is. 'T'en anderen so wanneer een dief oft moorder zijn tanden bebloct heeft, dat is te segghen, als hy daer zijn ghenuechte in ghenomen lieeft, soo en isser gheen groote hope van beteringhe in gheleghen.

(s. 19) Al is dat de Rechter somtijts clachten hoort ouer cenighe van sinen ondersaten, nochtans en sal hijer gheen haestighe vvrake ouer doen: maer sal hem regeren nae den Raet ende segghen van zijne vijise ende goede Raetslieden, roepende den misdadighen tsijnen verantvvorden ende defeucien.

(s. 22, 10) De drvase en canmen niet beter bedrieghen, dan met prijsen ende sulcke ghiften gheuen als sy gheerne bebben, of daerse meest ghenuechten in hebben. Den hooueerdighen verleytmen met tijtlijcke glorie, den gulsighen met spijse cnde dranck, den ghierighen met gout ende siluer, den luxurieusen met schoone vrouvven etc. hebbe einen vrunt bi deme heren, de ene vorantwordet in sineme afwesende.

(zu I, 4, s. 13, 6) .. dat nemant sineme viende loven schal to grunde .... dat he kumpt under eineme schine unde klede der geistlicheit efte hillicheit. . .

'To deme dridden male wert bir bewiset van den quaden, dat so wannêr ein morder, ein rover, ein vechter, de gerne blôt vorgeten, so wannêr ere tene sint blodich geworden, dat is, wannêr se hebben genochte efte en wol smekt quât to dôn, dat selden efte nummer men beteringe van den derf vormoden.

(zu I, 6, s. 16, 2) . . al isset so, dat ..... . richter waraftige klage horet van sinen undersaten ... dat he nochtans nene hastige wrake over em dôn schal. ... dat he hebben schal kloke wise radeslude, de wîsheit wetten, unde sake, dede lastich is, de in rechtverdicheit to underscheiden

(zu I, T s. 19, 4) .... dat de listige bedreger den dummen priset unde lovet

also den

hoverdigen doren mit tîtliker ere, den vrazigen mit spise unde dranke, den girigen mit gelde und gaven, den unkuschen mit vrouwen. 
h

(8. 32, 14) Die is sot die hem laetleyden in een onbekende plaetse daer de leytsman niet vorgaen en vilt.

(s. 3s, 21) Niemant en behoort hem tonderwinden te doene, tghenc dat sijn officie niet en is gelijck de Wolf die de clocken vvilde trecken. Ooc vvort hier den ghicrigen houelinck gheleert, dat hy niet soo veel en rape, dat hijer door in een alsulcken last en come, daermen niet lichtelijck vvt gheraken en can.

(s. S1, 13) Als de Vossen sien dat sy tvelt alleen hebben, ende dat niemant meer en elaecht, soo spreken sy stoutelijck.

(s. 98) Altijt sijnder int Hof ghier-vvolnen, die gheerne metten Prince deylen tot huerliedē voordele, maer alst de Vossen ter herten nemen, soo vinden sy hen bedroghen. (zu I, 12, s. 35,10$) \ldots$ desse is gek unde unklôk, de sik let leiden in umbekande stede, dar de leidesman nicht vorgân wil . .

(zu I, 17, s. 54, s) .. dat nemant vuldôn schal siner sinliken lust ... gelîk deıne wulve, de van lusten ludde de klockeu . . . . . . .

. . . . . . dar he van deme wulve secht, dat he so vele at, dat he ût dem gate nicht wedder konde komen sat, dar he hungerich in quan

(zu III, 4, s. 159, 4) .. dat de quade, de besecht is, desse, wan he sut, dat de simpele vorbluffet wert unde sine klage nicht vorvolget, desse sprikt denne gerne kônliken ....

(zu III, 13, 8. 183, 2) ... dat erste is, wo etlike girige, untruwe vogede in der heren hove vor sik de besten morsele beholden .......

Nach dem gesagten ist es selbstverständlich, dass wir an vielen stellen im R. V. nl. vorlage baben, wo ein directer hinweis auf eine nl. recension dies nicht beweisen kann: das eine ist aber zur sicherheit gowordon, dass die selbständigkeit des nd. ubersetzers auch für die glossc gelcugnet werden muss.

Damit kommen wir auf die zweite frage, in wie weit R. V. zusätze gemacht hat und ob wir dieselben erkennen können. - Sehen wir, ob sprachliche eigentumlichkeiten uns nach dieser richtung eine handhabe bieten. Latendorf a. a. o. s. 33 sagt 'dic ganze sprache der glosse weist fur nicht wenig partien auf einen nl. ursprung unverkennbar hin'; er scheint hierbei, wie seine folgenden ausführungen dartun, vor allem 
an einzelne entlehnte wörter zu denken, worauf ich gleich zurïckkomme.

Was zunächst die wortfïgung betrifft, so bat die nol. glosse in derselben manche eigenheiten (vgl. auch Libben s. $24 . \mathrm{zu}$ gl: I, 17, s. 56, 18); allein eine zusammenstellung derselben hat ergeben, dass ähnliche constructionen wie verbindung cines im singular stehenden verbums mit einem substantiv im plural und umgekehrt, eines accusativs mit dem infinitiv, eines doppelten accusativs bei laten, oder aus vorhergehenden wörtern zu ergänzende substantiva oder verba, anakoluthien, pleonasmen u. s. w. in allen germanischen sprachen vorkommen, so dass die glosse des R. V. zu den von Kosegarten in Höfers Zeitschrift fur die Wissenschaft der Sprache Bd. I-III gesammelten syntaktischen beobachtungen im mud. zwar manche hubsche parallelstellen, für die uns beschäftigende frage jedoch keine ausbeute gewährt.

So wenig wir also in syntaktischer bezielung anhaltspunkte haben, blosse übertragungen aus dem nl. von zusätzen des tibersetzers zu unterscheiden, so wenig wird es uns meiner ansicht nach gelingen, dafur ein kriterium aus der verwendung von fremdwörtern zu gewinnen.

$\mathrm{Zu}$ diesen hat Latendorf a. a. o. s. 33 auch 'merje' und 'genochte' gerechnet; ersteres ist im mnd. wb. nur aus unserm werke belegt; 'genochte' aber, was in der glosse des R. V. an folgenden stellen 8. 13, 3; 13,5;13,12;29,17;96, 1; (vgl. genôchlik \&. I, $1 \delta ;$ VI, $3 ; 95,17$ ) vorkommt, war dem ubersetzer geläufig, worauf schon Latendorf binweist, da es in der zweiten ausgabe des nd. Narrenschiffs von 1519 I, 43 vorkommt; da dicses aber auf beeinflussung durch $R$. V. beruhen könnte, so sei bemerkt, dass schon die erste aus derselben druckcrei wie R. V. hervorgegangene bearbcitung des Narrenschiffes, Llibek $1497^{1}$ ), an dieser stelle dasselbe wort hat.

1) Das einzige von Zarncke, Lit. Centralbl. 1867 s. 104 nachgewiesene exemplar im britischen museum hat 237 bezifferte bll.; auf der voll bedruckten seite stehen (wie in R. V.) 22 zeilen; das ende ist defect. Die typen sind dieselben wie diejenigen des $R$. V., ebenso befinden sich auf den den erdboden darstellenden partien der bolzschnitte dieselben eigentümlichen zeichen ...... wie anf den holzschnitten des R. V. Diese mitteilungen verdanke ich der güte des herrn dr. R. Hörning in London. 
Nicht besser steht es mit den fremdwörtern der glosse, von denen Latendorf a. a. o. eine anzahl zusammengestellt hat; ich fiihre sie im folgenden alle auf: artikel s. 44, 31; benediginge s. 92,5 ; capittel s. IV , 6 ; IV, $8 ; 9,1$; 13,1; 16, 1 ; u. ö.; cappelân s. 92,9 ; 132, 16 ; kastien s. 55,30 ; complexie s. 116,10 ; contrarie s. 108, 9 ; (mede-) kumpân s. 56, 11 ; deken s. 13,9 ; exempel s. III, 19; fabele s. III, 11; III, 15; III, 17; 188,$14 ; 191,10 ; 203,6 ; 221,2 ; 226,2 ;$ verse s. III, 14; 189, 5; historie s. III, 10 ; III, $17 ; 170,4 ; 205,2$; ypocriserie s. 34,2 ; 58,11 ; materie s. 170,$5 ; 170,6 ; 196,12$; morsêl s. 183,3 ; ordinancie s. 172,7 ; pape s. 43,$30 ; 43,38 ; 44,13 ; 137,10 ;$ part s. IV, 6 ; IV (überschr. z. 2. vorrede); pelegrim s. 95,2 ; pelegrimacien s. 92,3 ; peuitencie s. 56,22 ; philosophi s. III, 8 ; pinsen s. 67,2 ; poete s. III, 10; 20, 2; 29, 2 u. ö.; prelate s. 93,$5 ; 131,7 ; 131,9 ; 131,11 ; 137,2 ;$ provene s. V, 27; 9, 4; 54,$17 ; 54,24 ; 54,25 ; 137,30$; provest s. 93,8 ; profete s. 29,23 ; 137,20 ; profit s. 108,$13 ; 160,34 ; 170,7$; profitelik s. 170,6 ; regiment s. 9,8 ; regul s. 43,30 ; rente s. 54,18 ; reverencie s. 63,3 ; salm s. 29, 24; simpel s. 101,$3 ; 101,4 ; 108,5 ; 150,2 ; 150,6$; 160, 2 ; subtil s. 17,$4 ; 48,2$.

Wenn wir dem gegenuber die beobachtung machen, dass an manchen stellen den fremdwörtern im $\mathrm{nl}$. texte deutsche ausdricke im $R$. V. entsprechen, wie: $h, 22,13$ glorie $=r, 19,7$ ere; h, 22, 15 luxurieusen $=\mathrm{r}, 19,8$ unkuschen; h, 57, 13 palster $=\mathrm{r}$ uberschrift $\mathrm{I}, 33$ staf; $\mathrm{p}, \mathrm{LXV}$ serpent $=\mathrm{r}$ uberschrift III, 4 lintworm efte slange; h, 100, 1 gheaccuseert $=\mathrm{r}$ uberschr. IV, 1 klaget; $\mathrm{h}, 106,18$ presenteert $=\mathrm{r}$ uberschrift IV, 5 bôt; so möchte es auf den ersten blick scheinen, als ob wir in denjenigen partien, in denen sich im R. V. fremdwörter finden, spuren des originals erhalten lïtten. Allein dagegen muss gesagt werden, dass einmal das volksbuch, gemäss der entwickelung des nl., uberhaupt grosse neigung zu romanischen ausdrücken den ubrigen recensionen gegenüber verrät, andrerseits aber auch die nd. sprache dieser periode von fremdwörtern nicht frei ist, vgl. z. b. das nd. Narrenschiff von 1519 in Zarnckes ausgabe (ich führe nur solche stellen an, wo hertibernahme aus dem hd. ausgeschlossen ist) zu 61 processie; zu 34 absolueret; zu 65 practyken; zu 103 geprent, conscientien; zu $110^{\mathrm{b}}$ collacien, lectie lesen, lexie; ferner vorrede v. 106 
profit; contraric 1,$66 ; 2,68 ; 4,144$; materie 1,97 ; temptatien 4,36 ; regiment 5,35 ; testament 5 , 36; firmament 5,112 u. s. w.

Da dies also zur erkennung von zusätzen in der glosse nicht ausreicht, so mussen wir uns nach andern gründen umzusehen versuchen. Ganz sichere, wie z. b. bezugnahme auf specifisch nd. einrichtungen oder anspielung auf Lubek und umgebung oder gar nennung des namens des glossators finden wir nicht: was ich für den nd. libersetzer beanspruche hat daher nur bis zu einem gewissen grade wahrscheinlichkeit für sich.

Um mit dem relativ sichersten anzufangen, so scheint mir ist daran kein zweifel erlaubt, dass 8. 142, 1 Van dessen secht he nicht vele in desser allegginge mit dem he der nl. glossator gemeint sei; ich halte daher die eigentliche glosse 141,1-142, 1 fur original und die worte 142,1 bis ende fur zusatz des nd. - Dasselbe gilt von der glosse z. III, 12 s. 179. Up dit vorgesechte capittel is sunderlik nene ûtlegginge gesat etc. und von s. 91, 1 ff. und 116, 1 ff.: In desseme capittel is nicht sunderlikes, doch mach men hir inne merken twei stucke: hier ist der eingang zu sehr abweichend von dem sonstigen verfahren, wo immer ganz bestimmt gesagt wird, so und so viele stlucke kann man lernen, während es hier durch die worte doch mach men gewissermassen dem leser uberlassen bleibt, dies als moral hinzunehmen oder nicht.

Wegen einer ähnlichen abweichung vom gebrăuchlichen, nämlich der anknlupfung mit $\hat{o} k$ is de meiste $\sin$ s. 132, 17, nachdem schon vorher die 6 stucke abgetan sind, lege ich die stelle s. 132, 17-19 dem niederdeutschen bei und das durchbrechen der s. 89, 1 zusammengefassten vorhergehenden 7 capitel durch die glosse 8. 83, 1 Hir na wert gesecht, alse wan ein untruwe schalk bi einem vorsten is belastet etc., die zudem nicht wie gewöhnlich das vorhergehende glossiert, sondern den blick nach vorwärts richtet, wird auch nicht im original gestanden haben.

Die glosse zu IV, 5, s. 205 weicht im inhalt ganz auffallend von den tibrigen $a b$, indem hier, während sonst aus dem inhalt des betreffenden vorhergehenden capitels eine moralische lehre gezogen wird, sich die widerholung einer kampfessitte breit macht, die durch den text schon hinlänglich 
geschildert war und welche die uberschrift $\mathrm{zu}$ IV, 5, s. 203 sowie die inhaltsangabe s. 188, 7 auch schon erwähnt hatten; der verfasser vergisst auch nicht seiue gelebrsankeit auszukramen und zu zeigen, dass er in römischer und anderer ge. schichte ein belesener mann sei. - Eine ähuliche glosse zu I, 12 , s. 35,8 könnte man, da doch s. 34,1 nur von 8 stucken die rede ist, gleichfalls hierher rechnen, doch dem stebt entgegen, dass h $\mathrm{s} .39$ cinen teil dieser partie als moralisation hat; hiernach kann man vermuten, dass die originalglosse schon einmal im nl. erweitert worden ist. In der schlussglosse s. 226 gibt sich, däucht mich, derselbe ungeschickte nd. verfasser zu erkennen, der so unbeholfeu das gespräch 1) mit dem affen v. 4097 aus dem zusammenhange herausriss und uns dafür dreimal (v. 4094, 4233, 4235) die versicherung gibt, dass Grimbart und Reinke am hofe angelangt seien: - hier schwächt der schluss, der alles dem geneigten leser viberlässt, den anfang doch zu sehr ab, wo mit ernsten worten die notwendigkeit der glosse hervorgehoben wird. Diesem steht nicht entgegen, was der glossator s. VI, 23 sagt, wo die worte nicht auf die glossierung bezogen $\mathrm{zu}$ werden brauchen.

$\mathrm{Zu}$ einer andern kategorie von erweiterungen kann uns die bemerkung fuhren, dass im text. des $R$. V. die anreden an die zuhörer sammt und sonders getilgt sind: ich vermute, dass sie es auch schon in der vorlage waren, denn weshalb hätte der ubersetzer sie im texte fallen lassen, während er sie in der glosse (8. 20, 17 so gi horen scholen; vgl. 8. 83, 6 so gi hir na mogen horen van deme hasen) gebraucht, und an der stelle der vorrede, die wir oben s. 26 dem niederdeutschen zuwiesen (s. VI, 3 umme dat desto nôchliker sî deme leser unde tohorer; s. VI, 22 welkere worde men horen unde lesen mach) ausdrücklich von zubörern spricht? Wie er hier und an folgendeu stellen: s. VI, $26 ; 17,7 ; 20,7 ; 20,17 ; 20,26 ; 30,4 ; 44,36$; 63,$14 ; 67,4 ; 70,20 ; 83,1 ; 83,7 ; 89,18 ; 89,30 ; 96,7 ; 102,10 ;$ 110,$5 ; 205,14 ; 220,8$; seine blicke nach vorwärts schweifen lässt, so wendet er sie auch oft genug zurlick: s. 29, 16;34, 22; 44,$24 ; 54,31 ; 59,4 ; 161,1 ; 115,20 ; 117,2 ; 173,5 ; 188,2$;

1) Anders urteilt hierüber: Knorr, Reinaert de Vos und Reinke Vos. Progi. d. Gelehrtenschule z. Eutin. Eutin 1857 s. 64. 
ja es werden sogar zweimal (s. 42, 5; 44,1) mit penibler sorgfalt die blattzahlen genaunt, auf welchen ähnliches vorkommt - dies stimmt wenig zu der korten iutlegginge des Hinrek van Alckmer, wol aber zu de! grossen brcite des nd. ubersetzers, der wir schon öfters begegueten. Eine wesentliche stütze gewinnt dic erste stelle s. 20, 17 dadurch, dass, während s. 19, 1 von nur 3 stiicken gesprochen wird, doch s. 20, 7 mit $\hat{o k}$ ein neues stiick hinzukommt, dessen breite theologische ausführung grund genug ist, es dem Niederländer abzusprechen.

Als theologisch gebildet lernten wir den nd. übersetzer auch schon oben s. 27 kennen, wo wir ihm die vorrede zum vierten buche glaubten zuweisen zu müssen und ebenfalls tritt an den stellen, wo von geistlichen einrichtungen und den schïden derselben gesprochen wird s. 34,$7 ; 43,28 ; 54,17-56,19$; 131; 137 seine geistliche bildung zu tage. Sicher können wir hier die glossen s. 131 und 137 dem Niederdeutschen beilegen, da der text vv. 3829-4096, auf welchen sich die glosse bezieht, selbständig umgearbeitet ist; auch die dieser ähnliche glosse s. 43, 28 zeigt in ihrer ganzen ausfuhrung mit der lebhaften interjection $\hat{o}$ wô quâtliken, mit der anknlipfung durch $\hat{o} k(s .44,21 ; 44,26 ; 44,29) \mathrm{mit}$ dem directen hinweis auf eine frübere blattzahl (s. 43,28) höchst wahrscheinlich nl. vorlage ab - und in s. 34, 7-19 gibt sich ein so energisch ausgedrückter unwille gegen das festhalten an heidnischem aberglauben zu erkennen, dass man diese stellen als zusatz anzusehen allen srrund hat. Und müssen wir, angesichts der uber eine seite sich erstreckenden ausführlichen, mit belegstellen aus der bibel durchflochtenen darstellung s. 54, 17-56, 9 uber diese glosse nicht ein gleiches urteil fällen? - Endlich bleibt noch von diesen stellen s. 89,18 ubrig, wo die selbst:ïndigkeit des übersetzers klar ist, da die glosse eigentlich rhon zu ende ist und nur noch einmal zu grösserer bekräftirung der dritte punkt in form von beweisen aus dem alten tcistament widerholt wird. - Hierdurch habe ich schon meine : Insicht ausgesprochen tiber die citate und weise also folgende lem Niederdeutschen zu: s. 20,22;34, 17;43, 23;44, 4; 51, 27 ; $5.5,5 ; 55,10 ; 55,27 ; 89,20 ; 89,24 ; 137,13 ; 137,20 ; 137,34$; 189,$1 ; 189,4$ und das sprichwort 91,3 ; nebst diesen bezieht sich die glosse noch an folgenden stellen auf die bibel: s. IV, 
$\left.\left.17 ; 29,8 ; 29,23 ; 59,2^{1}\right) ; 70,8 ; 93,6 ; 95,4^{1}\right) ; 95,12 ; 102,1$; 186,$15 ; 220,12$ und das sprichivort 141,3 ; bei diesen wird sich schwer eine sichere entscheidung treffen lassen, doch möchte man wegen vorliebe des übersetzers für citate geneigt sein, ihm auch einige von diesen zweifelhaften stellen zuzuschreiben.

Sicher nd. ist die glosse, die s. 42,11-43, 28 aus der erzählung des abenteuers des fuchses mit der wölfin gezogen wird, denn keiner der bekannten nl. texte überliefert diese episode.

Mit ähnlichen frischen, das verkehrte treffend strafenden worten wird s. $160,6 \mathrm{f}$. gegen die torheit in erfindung unsinniger moden geredet. Ich kann mir nicht denken, dass der Niederländer mit seiuer trocknen moral dies sollte geschrieben haben, zumal da ja der text auch nicht im geringsten anhalt zu solchen auslassungen bietet; wol aber ist mir glaublich, dass unser nd. übersetzer, der doch olne zweifel eben erst von der ubersetzung des Narrenschiffes herkam, in gerechtem eifer gegen die modenärrinen herzog, die er so trefflich von Brant hatte schildern und strafen hören, und unsere stelle schrieb in anlehnung an das vierte capitel ('Van nyen vunden') des Narreuschiffes, wo es in der nd. übersetzung von 1497 heisst:

Untuchtige vrau wen van lichten dingen

Vele boser sede se ok upbringen

De erbaren volgen dessen mede

Unde prysen syk desser quaden sede

So vele gudes men en nicht kan lesen

Se wyllen al dorynnen mede wesen

Ere wangen se malen, ere antlaet sueren

Ere haer voranderen, er vorhouet scheren

Edder laten yd syk myt pine vth plucken. etc.

1) Die nd. bibel, welche 1494 in Lüibek bei Steffen Arndes gedruckt wurde, scheint dem nd. übersetzer unbekannt gewesen zu sein; wenigstens lauten hier die stellen: 59, 2: nument de dar legghet sine hand an de ploch vin suet achler sik. is ghesclicket to deme ryke gades. Und 95, 4: wēte inwēedich sint se gripéde wulue Vä eré vruchtēe bekennet se. enlesen se vä dé dornē de druué. vä vä den dislelen de vigë. Andere stellen stimmen besser, so s. IV, 17, wo nur statt schaltu verstu und s. 48,4 wo statt minschen luden steht. 
Aehnlich kann man in der glosse s. 10, 5 anklänge finden an Henselînbôk (hrsgbb. v. Chr. Walther, Jahrbuch des Ver. f. nd. Sprchfschg. III), (las später in der protestantischen glosse des R. V. mehrfach wörtlich ausgeschrieben wird und un dieselbe zeit wie R. V. in derselben officin gedruckt wurde: a. a. 0. s. 14 , v. 17 :

Beholt de arme wat, he [de rentner] neme dat wol dar to Nicht hir, men in den steden in Lomberdien,

Dar don se so, ya nemen wech beyde swyn unde ko. -

So haben wir gesehen, dass, wenn auch der nd. uibersetzer im grossen und ganzen für die glosse das nl. original zu grunde legte, er doch im einzelnen in nicht wenig punkten ganz wesentlich von demselben muss abgewichen sein; die bisher und besonders seit dem bekanntwerden des nl. gedruckten gedichts von Reinaert behauptete übereinstimmung mit der nl. glosse ist so wenig vorhanden, wie eine fruber allgemein angenommene selbständigkeit: wir sehen hier gewissermassen das vorbild für den spätern protestantischen glossator, von 1539 , der sich in ähnlicher weise von der katholischen glosse entfernt, wie der Lilbeker ubersetzer es von der niederländischen wird getan haben.

\section{Der erzählungstext des R. V.}

Wenden wir nach diesen betrachtungen den blick zuruck zum erzällungstext des R. V., so haben wir uns vor allem nach dem gedruckten Reinaert und seinem verhïltnis zum R. V. umzusehen. $d$ stimmt nun vers für vers mit b uberein, die inderungen in einzelnen, dic im allgemeinen von untergeordneter bedeutung siud, und meist nur dem ausdruck auflielfen wollen, werden für uns dadurch wichtig, dass folgende von ihmen mit $r$ gegen $b$ stimmen:
a, 1512 des haddic dicke goet ghelach
b, 1550 daer ic mi dicke op te saden plach
d, 34 daer hi ontfinc menighen slach
p, XX" daer ic mi dicke te sade plach
$r, 145 \mathrm{~s}$ dar he entfenk mannigen slach
[h, s. 38, 8 tilgt]
a, 1737 laet mi doch lesen II pater nooster
b, 1757 laet mi doch lesen een pr nr te trooster 
p, XXIII Laet mi doch spreken een pater noster

d, 97 Laet mi doch lesen een Pater noster

$r, 1671$ latet $\mathrm{mi}$ doch lesen ein pater noster

[h, s. 40 tilgt]

a, 1769 gheboot, hi gheve $u$, coninc heere langhe bliscap ende ere

b, fehlen diese verse, s. v. $17 £ 9$ Var.

p, XXIII" God wil $\mathbf{v}$ groteliken eren eñ vriēdelijcken groeten

d, 122/3 Gheboot die gheve u coninc heer

Langhe blischap ende groot eer

1, 1703/4 He sprak: eddele konink, gnedige here, dorch juwe eddelheit unde dorch juwe ere

h, s. 41,15 indert.

Diese gemeinsamen abweichungen führen uns dazu, dass $r$ nach d gearbeitet worden ist; der hauptberveis dafur ist der gemeinsame fehler $d, 34=r, 1458$; der vers ist hier ungehörig, während er einige zeilen vorauf $d, 28=r, 1452$ an seiner richtigen stelle stelit, wo ihn auch a, 1505; b, 1544; p, XX' haben. Aber auch $p$ steht näher zu $r$ als die tubrigen, da es mit $r$ vón der gewöhnlichen lesart abweicht.
a, 693
hi began brieschen ende bulen
hi was begrepen bi sier mulen
b, $7+5$
hi began te hulsen en te hulen ende mitten afstersten voet te trappen
$p, X$ rect. IX' hi begät te hulë en te brieschen eñ crassede metten afterste voetē
r, 643 He begunde to hulen unde to braschen
h, s. 25,12 mit den echtersten voten to kraschen Dewijle maecte Bruyne sulck getier
metten achtersten voeten

und besonders an folgender stelle:

b, 4038 op Westvalen ende te Provijn')

En gegaen tot hoger scolen mit ouden wisen sonder folen

p, LIII' mitt. Ic hebbe terfiorden ter scholen ghegaen

r, 3777 Hebbe ik doch to erfort de schole geholden

Es muss daher auch $d$ auf $p$ (oder vielmehr, da dieses als prosa-auszug nicht in betracht kommen kann, auf dessen

1) Martin, einltg. z. Reinaert.s. XXII müchte vermuten, dass auch ursprïnglich in b gestanden habe: te Westvalen op d' Erfortijn; doch gehörte Eirfurt nie zu Westfalen. 
vorlage $\pi$ ) zurlickgehen und der fehler $d, 34$ wird so zu erklären sein, dass das auge des verfassers auf den einige zeilen vorher vorkommenden vers abirte. Dies gereimte $\pi$, aus welchem eirerseits $\mathrm{p}$ den prosa auszug machte und aus welchem andrerseits d (und damit r) fliessen, gehörte selbstrerständlich zur recension R. II und gewährte in vielen fällen eine bessere thberlieferung als das erhaltene $\mathrm{b}$; man vergleiche a 255, r 259, b 266 var.: a $269 / 70$, r 281/2, b 296/7; a 299, r 305, b 327; a 448, r 426, b fehlt; a 482, r 460, b 512; a 590, r 575, b 644; a 705, r 662 , b $762 ;$ a 717 , r 671 , b $771 ;$ a $863 / 4$, r 795/6, b 901 ; a $1007 / 8$, r $901 / 2$, b $1031 / 2$; a $1435 / 6$, r 1379/80, b $1481 / 2$.

Auch stand es näher zu c als zu b:

b, 7373 alle die beste bliven di u Reinaert sprac danc hebben sie nu

c (var. zu b, 7373) alle die meeste bliven a bi Reinaert spranc danc hebben si

r, 654i alle de besten blivens ju bi Reinke sprac dank hebben se

$p$, CIl' Alle die beesten die gheuen $v$ des prijs daer van diet ghesien hebben Reinaer sprac des moetē si dāc hebbē.

Dass $\pi$ und c identisch gewesen seien, wäre eine vermutung, die wegen des zusammentreffens der jahreszahlen der herstellung von $p$ (1479) und e (1477, s. Martin, einltg. s. VII) ausgesprochen, aber nicht bewiesen werden kann.

$\mathrm{Zu}$ diesem resultate, welches nur aus der betrachtung der texte entstanden ist, stimmt vortrefflich die vermutung H. Bradshaws, dass d im jahro 1487 bei G. Leeu in Antwerpen gedruckt wurde. Es wurde dann von der gleichen druckerei der prosa-auszug 1479 und der gereimte und glossierte Reinaert 1487 ausgegangen sein, indem man zur herstellung des letzteren, mit welcher Hinrek von Alckmer beauftragt wurde, $\pi$ und daneben das schon mit einer kurzen einleitung sowie capiteleinteilung versehene $p$ benutzte; - ob dann später vielleicht d einer nochmaligen revision in bezug auf weitere glossierung und capiteluberschriften unterzogen wurde, und erst hieraus $r$ entstand, mag dahin gestellt bleiben.

LEIPZIG, im sept. 1880.

FR. PRIEN. 